\title{
Comprehensive genome-wide analysis of calmodulin-binding transcription activator (CAMTA) in Durio zibethinus and identification of fruit ripening-associated DzCAMTAs
}

Zahra lqbal', Mohammed Shariq Iqbal', Lalida Sangpong ${ }^{1}$, Gholamreza Khaksar ${ }^{1}$, Supaart Sirikantaramas ${ }^{1,3}$ and Teerapong Buaboocha $a^{1,3^{*}}$

\begin{abstract}
Background: Fruit ripening is an intricate developmental process driven by a highly coordinated action of complex hormonal networks. Ethylene is considered as the main phytohormone that regulates the ripening of climacteric fruits. Concomitantly, several ethylene-responsive transcription factors (TFs) are pivotal components of the regulatory network underlying fruit ripening. Calmodulin-binding transcription activator (CAMTA) is one such ethylene-induced TF implicated in various stress and plant developmental processes.

Results: Our comprehensive analysis of the CAMTA gene family in Durio zibethinus (durian, Dz) identified 10 CAMT As with conserved domains. Phylogenetic analysis of DzCAMTAs, positioned DzCAMTA3 with its tomato ortholog that has already been validated for its role in the fruit ripening process through ethylene-mediated signaling. Furthermore, the transcriptome-wide analysis revealed DzCAMTA3 and DzCAMTA8 as the highest expressing durian CAMTA genes. These two DzCAMTAs possessed a distinct ripening-associated expression pattern during post-harvest ripening in Monthong, a durian cultivar native to Thailand. The expression profiling of DzCAMTA3 and DzCAMTA8 under natural ripening conditions and ethylene-induced/delayed ripening conditions substantiated their roles as ethylene-induced transcriptional activators of ripening. Similarly, auxin-suppressed expression of DzCAMTA3 and DzCAMTA8 confirmed their responsiveness to exogenous auxin treatment in a time-dependent manner. Accordingly, we propose that DzCAMTA3 and DzCAMTA8 synergistically crosstalk with ethylene during durian fruit ripening. In contrast, DzCAMTA3 and DzCAMTA8 antagonistically with auxin could affect the post-harvest ripening process in durian. Furthermore, DzCAMTA3 and DzCAMTA8 interacting genes contain significant CAMTA recognition motifs and regulated several pivotal fruit-ripening-associated pathways.
\end{abstract}

\footnotetext{
* Correspondence: teerapong.b@chula.ac.th

'Molecular Crop Research Unit, Department of Biochemistry, Chulalongkorn University, Bangkok, Thailand

${ }^{3}$ Omics Sciences and Bioinformatics Center, Faculty of Science, Chulalongkorn University, Bangkok, Thailand

Full list of author information is available at the end of the article
}

C C The Author(s). 2021 Open Access This article is licensed under a Creative Commons Attribution 4.0 International License, which permits use, sharing, adaptation, distribution and reproduction in any medium or format, as long as you give appropriate credit to the original author(s) and the source, provide a link to the Creative Commons licence, and indicate if changes were made. The images or other third party material in this article are included in the article's Creative Commons licence, unless indicated otherwise in a credit line to the material. If material is not included in the article's Creative Commons licence and your intended use is not permitted by statutory regulation or exceeds the permitted use, you will need to obtain permission directly from the copyright holder. To view a copy of this licence, visit http://creativecommons.org/licenses/by/4.0/. The Creative Commons Public Domain Dedication waiver (http://creativecommons.org/publicdomain/zero/1.0/) applies to the data made available in this article, unless otherwise stated in a credit line to the data. 
Conclusion: Taken together, the present study contributes to an in-depth understanding of the structure and probable function of CAMTA genes in the post-harvest ripening of durian.

Keywords: Auxin, CAMTA, Durian, Ethylene, Fruit ripening, Transcriptional regulation

\section{Background}

Fruit ripening is a highly coordinated, complex, and programmed process that involves various physiological modifications, such as changes in color, texture, aroma, quality, and nutritional value [1]. Depending upon the mechanism of action during ripening, fruits are classified into climacteric and non-climacteric groups. Autolytic increase in ethylene biosynthesis triggers the ripening of climacteric fruits, such as tomatoes and apples. In concurrence, several ethylene biosynthesis and signaling genes had been reported to play crucial roles in fruit ripening [2-4]. In contrast, the ripening of non-climacteric fruits such as grapes and strawberries is an ethyleneindependent process $[5,6]$. The process of fruit ripening is tightly regulated by transcription factors (TFs) $[7,8]$. Fruit ripening is also affected by environmental conditions, such as biotic and abiotic stresses [9]. Additionally, calcium $\left(\mathrm{Ca}^{2+}\right)$ has been implicated in maintaining fruit firmness and delaying ripening $[10,11]$. Often, molecular mechanisms mediating plant responses to environmental and hormonal signals involve signaling cascades in which $\mathrm{Ca}^{2+}$ acts as a pivotal second messenger $[12,13]$. Perturbations in cytosolic $\mathrm{Ca}^{2+}$ levels are sensed and interpreted by an array of $\mathrm{Ca}^{2+}$-binding proteins that function as signal sensors $[14,15]$. Calmodulin $(\mathrm{CaM})$ is a well-characterized $\mathrm{Ca}^{2+}$ sensor, which is known to modulate target proteins such as TFs [16], including the well-known CaM-binding transcription activators (CAMTAs) $[17,18]$. CAMTAs, also known as signal responsive (SR) proteins [18] or ethylene-induced CaMbinding proteins (EICBP) [17] have been extensively characterized as the largest CaM-binding TF family [18]. The CAMTA proteins are reported to possess six functional domains [19], namely (i) CG1 domain, unique DNA binding sequence; (ii) NLS, nuclear localization signal to target the protein into the nucleus, (iii) TIG domain, implicated in non-specific DNA interactions, (iv) CaMBD, CaM-binding domain for the interaction of CaM with CAMTA; (v) ankyrin (ANK) repeat, contributing to protein-protein interactions; and (vi) IQ motif, region of low complexity for binding CaM and CaM-like proteins. CAMTAs were first reported as an early ethyleneresponsive $(N t E R)$ gene implicated in ethylene-regulated plant death and senescence, indicating its probable role in prolonging the shelf life of crops [18]. Later, a homolog of NtER1 and five related genes in Arabidopsis (AtSRs) were shown to play significant roles in several signal transduction pathways, including hormonal, developmental, and environmental cues [20]. Since then, CAMTAs have been reported to play significant roles in various biotic stresses, abiotic stresses, and plant development [21-24]. CAMT As are present in a number of eukaryotic genomes, including Arabidopsis thaliana [25], Oryza sativa [26], Solanum lycopersicum [27], Vitis vinifera [28], Medicago truncatula [29], Fragaria ananassa [30], Glycine max [31], Zea mays [32], Brassica napus [33], Populus trichocarpa [34], Gossypium hirsutum [24], Nicotiana tabacum [35], Citrus sinensis [36], Musa acuminata [37], Phaseolus vulgaris [38], and Linum usitatissimum [39]. The underpinning mechanisms of CAMTAs in drought, cold, and salt tolerance has been reasonably elucidated [23, 40-42]. Similarly, Arabidopsis thaliana CAMTA3 has been comprehensively studied for its involvement in biotic stress responses [22, 43, 44]. Additionally, the phytohormonal regulation of CAMTAs is extended to auxins [32, 45, 46], brassionosteroids [47], ethylene [27, 30, 48], abscisic acid (ABA) [32, 41], methyl jasmonate [32], and salicylic acid $[32,40,49]$. Apart from the notable involvement of CAMTAs in stress and hormonal biology, a unique involvement of Solanum lycopersicum CAMTAs (SISRs) in fruit development and ripening has been reported [27]. All seven SISRs were highly and differentially expressed during fruit development and ripening. The expression profile of SISRs significantly varied in rin mutant (ripening mutant) in comparison to the wildtype. Contradictorily, the alterations in expression of SlSRs were minor for the other two ripening mutants (nor mutant and $\mathrm{Nr}$ mutant). In addition, the treatment of mature green wildtype fruit with ethylene transiently enhanced the expression of all SlSRs [27].

To investigate the role of $\mathrm{Ca}^{2+}$-regulated CAMTAs in fruit ripening, we selected durian, a tropical fruit crop native to Southeast Asia. Durian is an economically important fruit that has recently gained huge popularity in international markets due to its unique odor, formidable spiny husk, and distinct flavor. It is considered an imperative export perishable item in Southeast Asia. Thailand is the top exporter of durian in Southeast Asia and other international markets. More than 200 durian cultivars are known; however, only a few of them are commercially cultivated and exported. In Thailand, four durian cultivars, Monthong (D. zibethinus Murr. 'Monthong'), Chanee (D. zibethinus Murr. 'Chanee'), Phuangmanee (D. zibethinus Murr. 'Phuangmanee'), and Kanyao (D. zibethinus Murr. 'Kanyao') are of great popularity in the local and international markets [50]. 
Monthong is a slow-ripening cultivar with less sweet taste, mild odor, creamy texture, and light-yellow pulp. It requires approximately five days after harvest (mature stage) to ripen. Once harvested, durian has a very limited shelf life. Hence, the ripening processes associated with durian is of great agronomic and economic significance for the agricultural industry. In Thailand, durian harvesting is performed at the mature stage (approximately 105 days for Monthong). Maintaining the flavor and nutritional value of durian fruit along with a longer shelf life poses a challenge for the industry. In neoteric times, research encircling the extended shelf life of fruits has gained momentum [51]. However, the underpinning mechanistic details fundamental to the fruit ripening process remain obscure. In addition, despite the fact that durian is gaining importance as an economic crop, genomic and molecular research on this crop is limited, especially with regard to the CAMTA TFs.

Coherently, the present study aimed to deepen our understanding of the structure and function of the CAMTA gene family in durian. This study identified ten DzCAMTAs in the durian genome. Comprehensive expression profiling of $D z C A M T A s$ during durian postharvest ripening revealed significant differential expression of $D z C A M T A s$ and proposed the putative ripeningassociated members. Our results evidently positioned $D z C A M T A 3$ and DzCAMTA 8 as the key members of the regulatory framework underlying the post-harvest ripening of durian. To our knowledge, the present study is the pioneer report examining the CAMTA gene family and its roles in the post-harvest ripening of this climacteric fruit.

\section{Results}

Genome-wide identification of durian CAMTA genes, their domain organization, gene structure, and motif composition

HMMER search was performed with the conserved DNA-binding CAMTA domain to identify CAMTA orthologs in durian species (Additional file 1-Table S1). After removal of redundant and partial sequences, 10 CAMTAs were identified in the durian genome (Table 1). Subsequently, all the putative CAMTA genes in durian were confirmed through similarity and conserved domain searches against the Pfam, NCBI conserved domain, and InterPro databases (Table 1 and Additional file 1-Table S2). The length of the identified durian CAMTA proteins varied from 891 amino acids to 1083 amino acids. The theoretical pI varied from 5.39 to 8.1, whereas the molecular weight ranged from 99,995.47 Da to $122,683.04 \mathrm{Da}$. The number of exons in $D z C A M T A \mathrm{~s}$ was quite similar (12-13 per gene), possibly indicative of functional redundancy. Additionally, all the identified $D z C A M T A$ s were presumably localized to the nucleus with a high degree of reliability (Table 1 ). This implies that the physicochemical characteristics of DzCAMTAs were highly conserved, with few obvious exceptions. For the standard annotation of the ten DzCAMTAs, we followed the numeric nomenclature system applied to AtCAMTAs. The MSA of DzCAMTAs by ClustalX and their sequence scanning using the Pfam, NCBI conserved domain, and InterPro databases revealed that all the DzCAMTAs were composed of the reported CAMTA domains (Fig. 1A and Additional file 2). All DzCAMTAs contained the signature CG-1 domain, IQ motif, CaMBD, and ankyrin repeat (Fig. 1A). The ten identified DzCAMTAs also contained a bipartite nuclear localization signal (NLS) at the N-terminus (within the CG-1 domain), suggesting that this domain might contribute to a signal that directs the import of DzCAMTAs to the nucleus. Moreover, with the exception of DzCAMTA10, the remaining DzCAMTAs comprised the TIG domain. It has been previously reported that Arabidopsis thaliana, Arabidopsis lyrata, Gossypium raimondii, Gossypium hirsutum, Gossypium arboretum, and Capsella rubella also encode non-TIG CAMTAs [24, 52]. The TIG domain originated first in the ancestors of the land plants. Mutation of some vital amino acids in the TIG domain rather than complete deletion of the TIG domain occurred in the non-TIG CAMTAs during the course of evolution [52]. Thus, non-TIG CAMTAs might contribute to the expansion of DzCAMTAs. The number of IQ motif in DzCAMTAs varied from 1 to 2 and were consistently found at the C-terminus of the protein (Fig. 1A). Nonetheless, the present study in durian validated the reported functional domains of CAMTA TF [19]. The evolutionary relationship among DzCAMTAs was deduced by constructing an ML phylogenetic tree with stringent bootstrap values (Fig. 1B). The DzCAMTAs were divided into two groups. Group 1 was further divided into two sub-groups (sub-group 1A and sub-group 1B). Similarly, group 2 was also divided into two sub-groups (sub-group 2A and sub-group 2B). In sub-group $1 \mathrm{~A}, D z C A M T A 3$ and $D z C A M T A 5$ shared a similar exon-intron organization (13 exons and 12 introns each); whereas in sub-group $1 \mathrm{~B}, D z C A M T A 2$ and $D z C A M T A 4$ shared a related exon-intron organization (12 and 13 exons respectively) (Fig. 1C). Intriguingly, sub-group 2B DzCAMTAs (i.e., DzCAMTA9 and $D z C A M T A 10)$ shared a strikingly high similarity in terms of their exon-intron organization and distribution pattern (13 exons and 12 introns of almost similar lengths) (Fig. 1C). The similar exon-intron organization of these DzCAMTAs might be an indication of their functional redundant behavior or their origin as paralogous genes with different functions. Sub-group 2A $D z C A M T A 6$ possessed the largest introns. Whether this particular gene exhibits a distinct function from the rest 


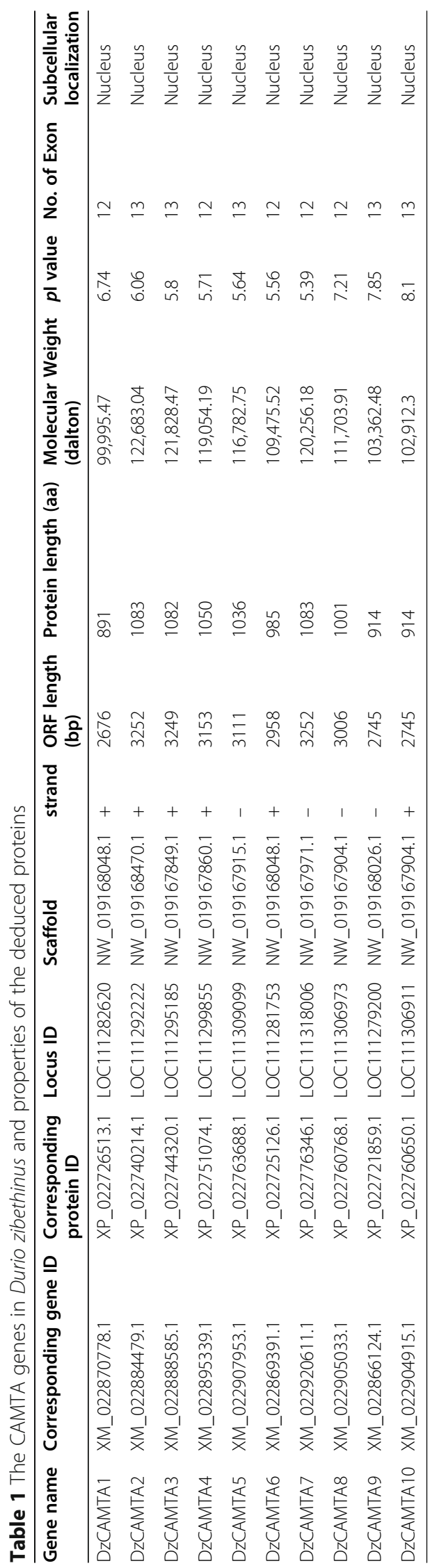




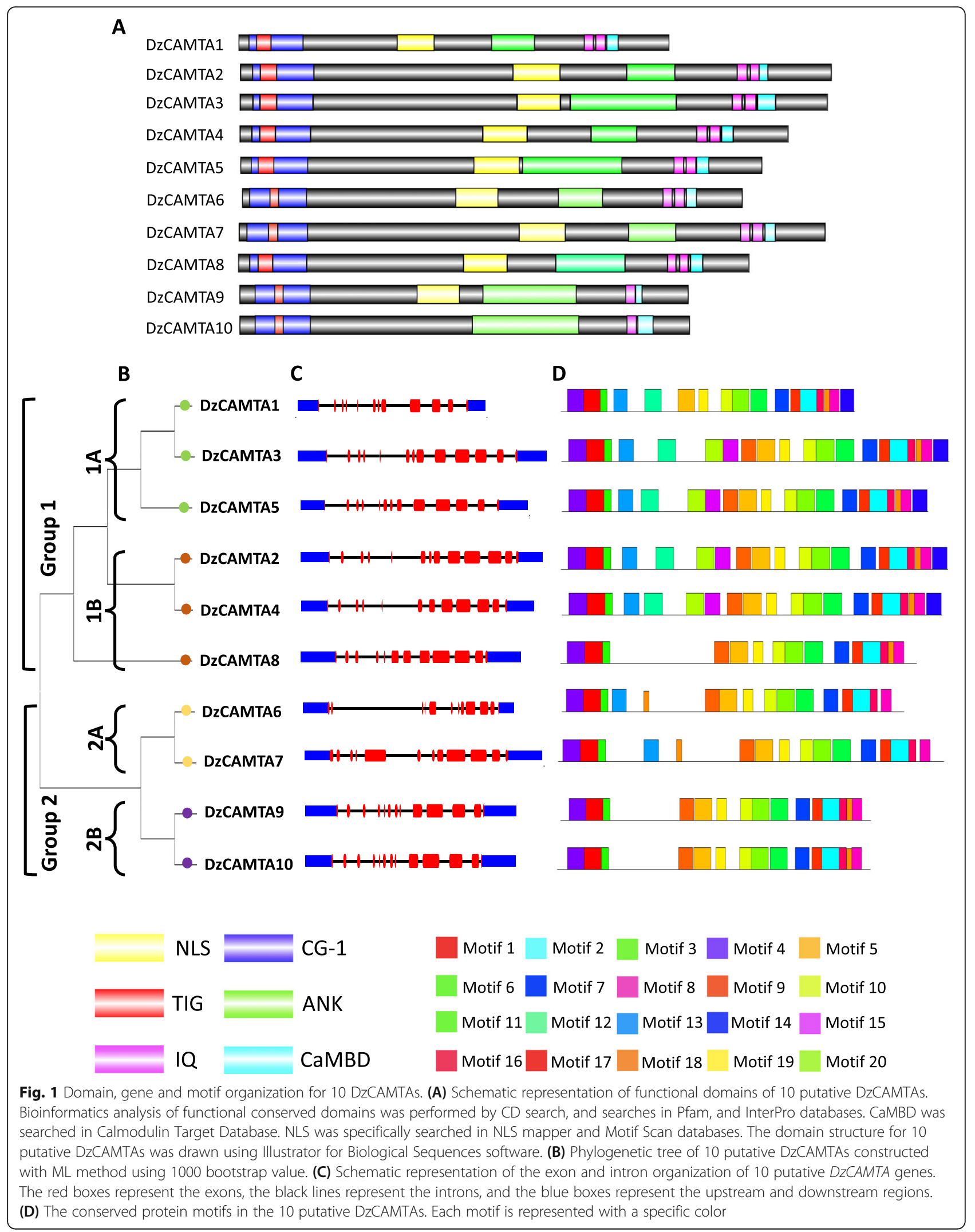


of the DzCAMTAs remains a subject for further study. Further, MEME tool was used to comprehend the motif conserveness among all $10 \mathrm{DzCAMTAs}$. Twenty putative conserved motifs were identified by MEME in DzCAMTAs (Fig. 1D and Additional file 3). Motifs 1, 4, and 11 that hit the Pfam, NCBI conserved domain, and InterPro databases were identified as CG-1 domain (Additional file 1- Table S3). Motifs 2 and 3 were the IQ motif and ankyrin repeat-containing domain, respectively (Additional file 1-Table S3). Motifs 5 and 9 were associated with the TIG domain (Additional file 1- Table S3). Motifs 1, 4, and 11 (the red, purple, and green motifs; CG-1 or the signature CAMTA motif) were identified in all the DzCAMTAs and represented the conserved CAMTA domain.

\section{Phylogenetic relationship of durian CAMTAs with other plant species}

A phylogenetic tree was constructed to gain insights into the evolutionary relationship of DzCAMTAs with CAMTAs from other plant species. The evolutionary relationships among the 10 identified DzCAMTAs and CAMTAs from four different plant species (Additional file 1- Table S4) were analyzed using the bootstrap consensus ML tree. We performed MSA of 10 identified DzCAMTAs with 36 CAMTA protein sequences from four different plant species (families: Brassicaceae, Solanaceae, and Malvaceae). Subsequently, the DzCAMTAs distinctly clustered with all the other four plant species with strong bootstrap support (Fig. 2). One interesting observation was the clustering of DzCAMTA1, DzCAMTA3, and DzCAMTA5 with Solanum lycopersicum Solyc01g105230 (SISR1L) that is well implicated in fruit ripening [27]. The DzCAMTAs and cotton CAMTAs (both plants belonging to the family Malvaceae) clustered together with very high reliability. This is indicative of the fact that DzCAMTAs and cotton CAMTAs originated from the same common ancestor. Consequently, it will be interesting to examine whether the CAMTA members of durian and Gossypium which clustered together function similarly, considering the involvement of Gossypium CAMTAs in fiber development [24].

\section{Scaffold distribution and duplication of durian CAMTAs}

Next, we assessed the physical locations of the $D z C A M T A$ genes in the durian genome. Since, only the draft genome is available for durian (organized up to scaffold level), the scaffold positions of $10 \mathrm{DzCAMTAs}$ were identified by BLASTN searches. The physical locations of each DzCAMTA gene are listed in Table 1. DzCAMTA1 and DzCAMTA6 (NW_019168048.1) as well as DzCAMTA8 and DzCAMTA10 (NW 019167904.1) were localized to the same scaffold (Fig. 3). The remaining six $D z C A M T A s$ were distinctly localized to separate scaffolds. We further assessed the gene duplication events responsible for the expansion of durian CAMTAs. Based on high protein sequence identity and similarity, five pairs of putative paralogous CAMTA genes were identified in the durian genome (Fig. 3). Intriguingly, the paralogous durian CAMTA gene pairs clustered together in the phylogenetic tree, depicting a high degree of protein sequence identity (> 70\%) (Fig. 2). It is worth mentioning that all five paralogous durian CAMTA gene pairs were located on different scaffolds. Moreover, the functional divergence and selection pressure of durian CAMTA genes were evaluated by Ka (nonsynonymous substitution), Ks (synonymous substitution), and $\mathrm{Ka} / \mathrm{Ks}$ (nonsynonymous by synonymous substitution) ratios between the paralogs. The $\mathrm{Ka} / \mathrm{Ks}$ ratios for paralogous durian CAMTA gene pairs were without exception < 1 (Additional file 1- Table S5). Hence, the duplicated durian genes had underwent strong purifying selection pressure accounting for the fact that they had not diverged much during the course of evolution. The duplication events for paralogous durian CAMTA gene pairs mainly occurred between 14.64 and 17.95 MYA (Additional file 1- Table S5).

\section{Durian CAMTAs encode CaM binding proteins}

The unique feature of the CAMTA TFs is the presence of the CaMBD (Fig. 4A). The existence of CaMBD has been reported in CAMTAs from all organisms except $C$. elegans [25]. Previously, it has been shown in Arabidopsis thaliana [25], Solanum lycopersicum [27], Fragaria ananassa [30], and Gossypium hirsutum [24], that CaMBD is composed of a functional motif (WXVX(2)LXKX(2)[LF]RWRX[KR]X(3)[FL]RX). This motif is considered indispensable for CaM binding [25, 27]. To determine the conservation of CaMBD, the CaM-binding regions of DzCAMTAs were aligned with Arabidopsis thaliana CAMTAs. We identified the conserved sequence of CaMBD as WSVG[IV]LEK[VA][IV]LRWRRK[GR] [SK]GLRG (Fig. 4B and C), which was consistent with previous reports $[24,25,27$, 30]. Thus, the amino acid composition of CaMBD in Arabidopsis thaliana CAMTAs shares a very high sequence homology with its durian counterparts. For example, DzCAMTA6 and DzCAMTA7 have almost the same amino acid composition as AtCAMTA4, DzCAMTA3 has almost the same amino acid composition as AtCAMTA1, and DzCAMTA2 has almost the same amino acid composition as AtCAMTA2 (Fig. 4B). Additionally, the CaM-binding motif is capable of forming an amphipathic $\alpha$-helix structure. We determined the amphipathic $\alpha$-helical properties of the 10 identified DzCAMTAs. All 10 DzCAMTAs formed an amphipathic $\alpha$-helix structure (for representation only DzCAMTA1 is shown). The results revealed an 18 

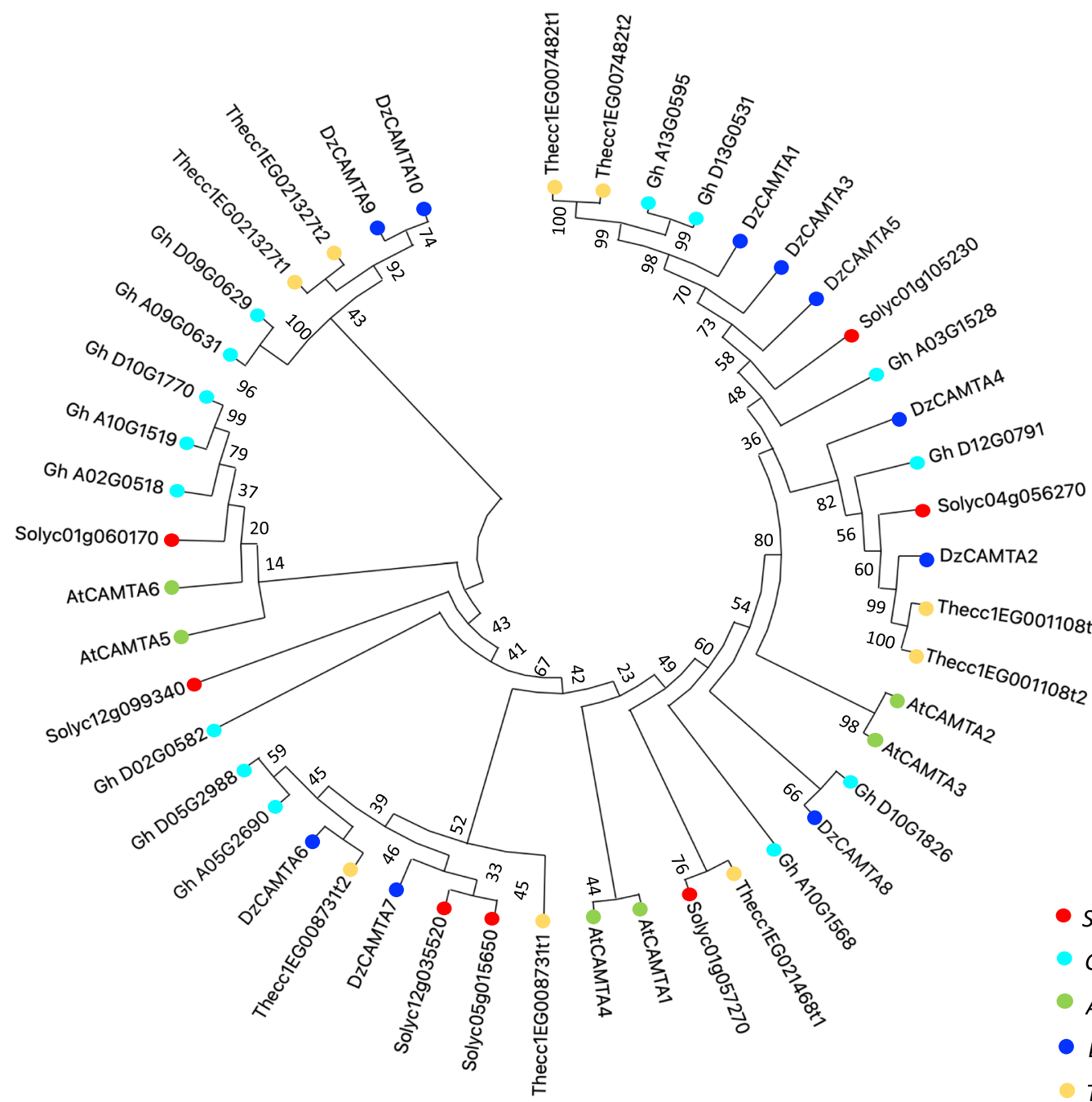

Solanum lycopersicum

Gossypium hirsutum

Arabidopsis thaliana

Durio Zibethinus

Theobroma cacao

Fig. 2 Phylogenetic tree of CAMTA gene family in Durio Zibethinus and four other representative plant species. The full-length protein sequences of Arabidopsis thaliana, Solanum lycopersicum, Theobroma cacao, Gossypium hirsutum, and Durio Zibethinus were aligned using MEGA X software. The phylogenetic ML tree was constructed with the JTT model and pairwise gap deletion using bootstrap test (bootstrap value $=1000$ ) with default parameters. The numbers against the subsequent branches indicate the bootstrap values that support the adjacent nodes. Different species are represented by colored dots; red: Solanum lycopersicum, aqua: Gossypium hirsutum, green: Arabidopsis thaliana, dark blue: Durio Zibethinus, and yellow: Theobroma cacao

amino acid long stretch to have a CaM-binding site (W, L, G, I, and V as the hydrophobic face) (Fig. 4D).

\section{Identification of cis-regulatory elements in the promoter of DzCAMTAs}

The identification of cis-regulatory elements in the promoter regions of the genes of interest provides important information regarding the gene function and regulation. The promoter sequences (1000 bp upstream to the transcription start site) of $10 D z C A M T A$ s were analyzed using the PlantCARE database [53] and the PLACE database [54] for the identification of cis-regulatory elements. Cis-acting elements implicated in abiotic and biotic stress, phytohormone regulation, and plant growth and development were identified in the promoters of the $10 \mathrm{DzCAMTAs}$ (Fig. 5A and B, Additional file 1- Table S6). The GT-1 motif was found in four DzCAMTAs (DzCAMTA1, DzCAMTA5, DzCAMTA6, and $D z C A M T A 9)$, which has been extensively linked to salt and osmotic stresses in Arabidopsis thaliana [55]. Similarly, G-box, which is well linked with bHLHs and bZIPs [56] TFs, was also found to be enriched in the promoters of six DzCAMTA genes (DzCAMTA2, DzCAMTA3, DzCAMTA4, DzCAMTA6, DzCAMTA7, and $D z C A M T A 8)$. It has been previously reported that the G-box is involved in the regulation of plant stress 


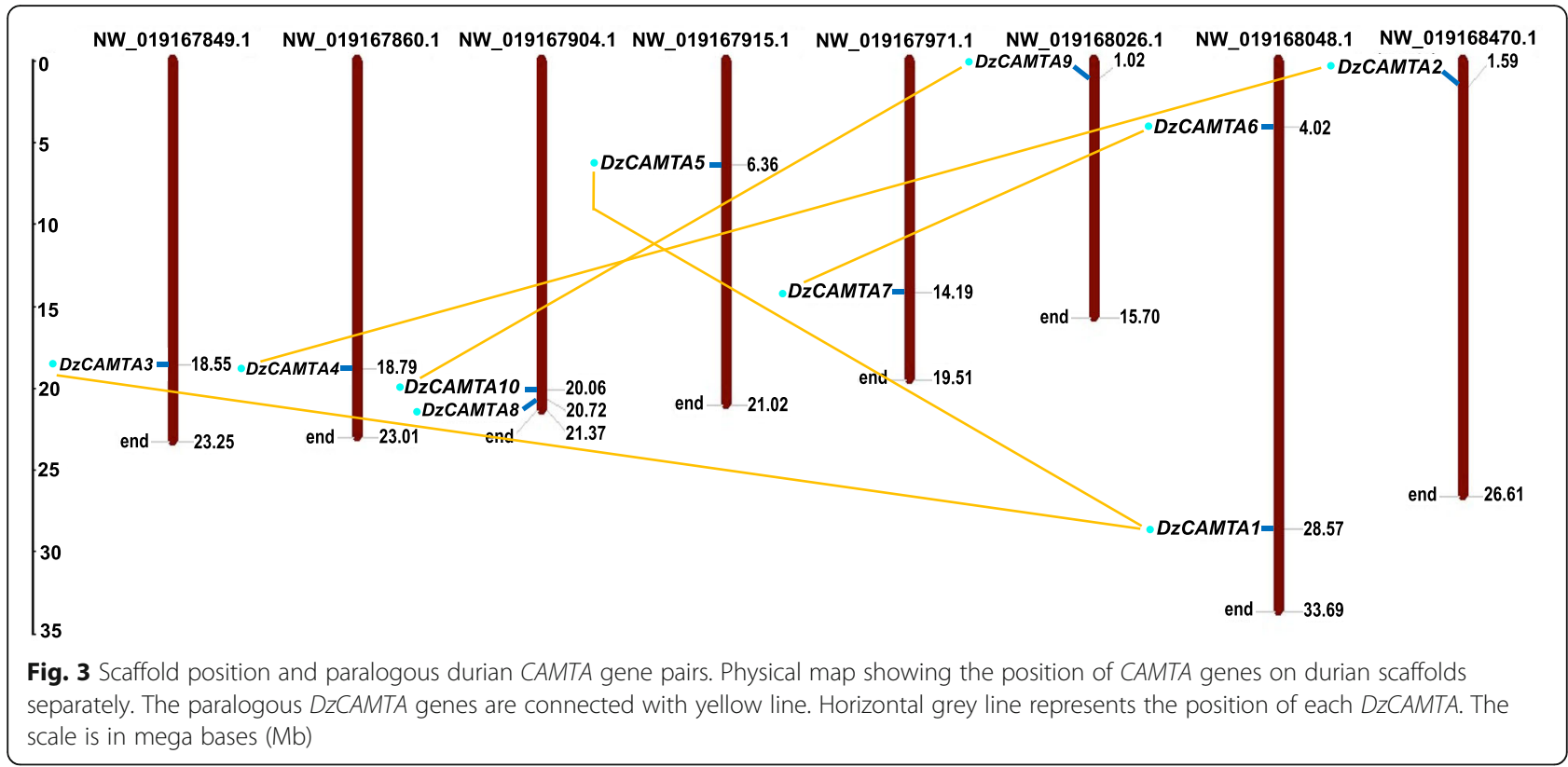

responses and early leaf senescence [56, 57]. Furthermore, it was interesting to note the presence of a few light-responsive cis-elements (LREs) in almost all the $D z C A M T A s$. These included GATA-box (DzCAMTA3), Gbox (DzCAMTA2, DzCAMTA3, DzCAMTA4, DzCAMTA6, DzCAMTA7, and DzCAMTA8), TCT motifs (DzCAMTA1, DzCAMTA3, DzCAMTA6, and DzCAMTA10), Box-4 (DzCAMTA1, DzCAMTA2, DzCAMTA3, DzCAMTA4, DzCAMTA7, DzCAMTA8, and DzCAMTA10), and chsCMA1a (DzCAMTA3 and DzCAMTA9) (Fig. 5A and B). This is probably indicative of the intersecting impact of light on the fruit ripening process during post-harvest handling $[58,59]$. However, promoter sequencing can provide a better and more reliable information regarding the binding sites. Phytohormonal cis-acting elements implicated in the regulation of MeJA-responsive element - CGTCA motif and TGACG motif [60], SA-responsive element TCA element [61], and ABA-responsive element - ABRE [62] were found to be enriched in the promoters of 8,3 , and $6 D z C A M T A$ genes, respectively. The gibberellinresponsive element- GARE motif, TATC box, and P-box [63] were present each in only one DzCAMTA gene. Similarly, the auxin-responsive element- AuxRR-core and TGA element [64] were observed in 1 and $3 D z C A M T A$ genes, respectively. As expected, the ethylene-responsive element (ERE) [65] was highly enriched in a high number of DzCAMTA genes (DzCAMTA1, DzCAMTA3, DzCAMTA4, DzCAMTA5, DzCAMTA6, DzCAMTA8, DzCAMTA9, and $D z C A M T A 10)$ (Fig. 5A and B). This indicates the probable cross-wired mesh of ethylene signaling in fruit ripening processes [4] through the involvement of DzCAMTAs. Considering the cis-acting elements in plant growth and development, MYB binding sites were found in almost all the
DzCAMTAs (DzCAMTA1, DzCAMTA2, DzCAMTA3, DzCAMTA5, DzCAMTA6, DzCAMTA7, DzCAMTA8, and $D z C A M T A 10)$ while the GCN4_motif involved in endosperm expression [63] was enriched in DzCAMTA9. The leaf development-related cis-acting elements HD-Zip1 [66] and the zein metabolism regulation element $(\mathrm{O} 2$ site) were present in DzCAMTA2 and DzCAMTA5, and in $D z C A M T A 4$ and DzCAMTA8, respectively. Additionally, CAT-box (meristem expression-related) was present in $D z C A M T A 1$ and DzCAMTA7, and MSA-like motifs (cell cycle regulation-related) was present in $D z C A M T A 5$ (Fig. $5 \mathrm{~A}$ and $\mathrm{B})$. Perhaps, the presence of these motifs is just indicative of the probable molecular regulation which can be further affirmed through sequencing.

\section{Differential expression of DzCAMTAs during post-harvest ripening of durian 'Monthong'}

We analyzed the expression pattern of the $10 \mathrm{DzCAMTAs}$ at three stages of post-harvest ripening, namely mature, mid-ripe, and ripe by qRT-PCR (Fig. 6). Notably, 8 out of $10 \mathrm{DzCAMTAs}$ were significantly expressed in fruit pulp and exhibited a ripening-associated pattern. These eight DzCAMTAs (DzCAMTA2, DzCAMTA3, DzCAMTA5, DzCAMTA6, DzCAMTA7, DzCAMTA8, DzCAMTA9, and $D z C A M T A 10)$ were considered as putative ripeningassociated TFs. DzCAMTA2, DzCAMTA3, DzCAMTA7, $D z C A M T A 8$, and DzCAMTA10 were up-regulated during post-harvest ripening. In contrast, the transcripts for DzCAMTA5, DzCAMTA6, and DzCAMTA9 were downregulated over the course of post-harvest ripening. $D z C A M T A 1$ and DzCAMTA4 did not show any significant changes in the expression levels. Since the expression levels of DzCAMTA1 and DzCAMTA4 did not 


\section{A}

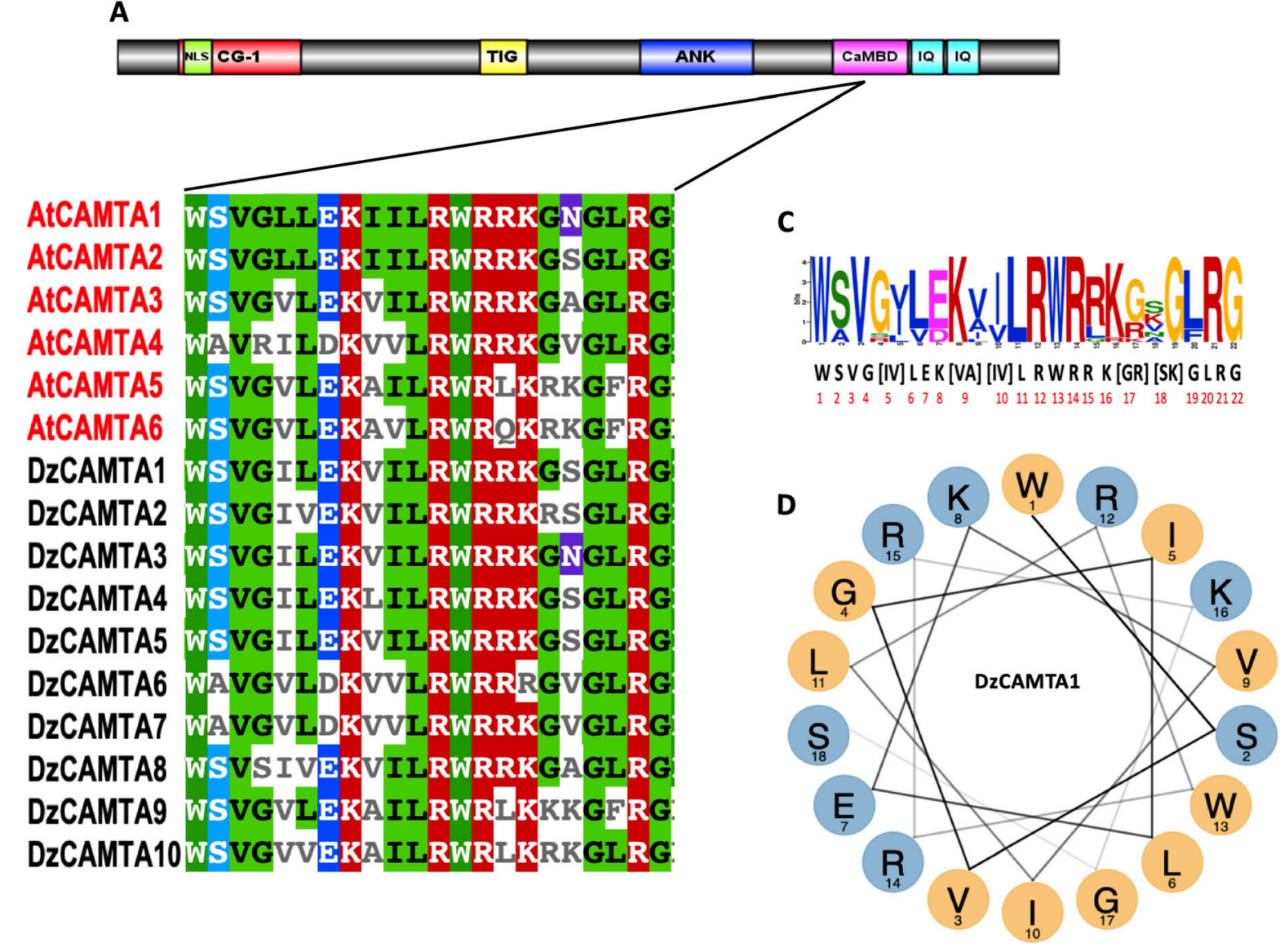

Fig. 4 Conservation of calmodulin-binding domain (CaMBD) in 10 putative DzCAMTAs. (A) General schematic representation of domain organization of CAMTA protein. (B) Alignment of the putative conserved CaMBD of Durio Zibethinus with CaMBD of Arabidopsis thaliana. (C) Logo for the sequence of CaMBD in 10 putative DzCAMTAs with 6 Arabidopsis thaliana CAMTAs. (D) Helical wheel projection of the representative amphipathic a-helix structure in the predicted CaMBD of Durio Zibethinus CAMTA1. The non-polar amino acids (G, L, W, I, and V) are shown by yellow circles. The polar amino acids (S, E, R, and $K$ ) are shown by blue circles. W, L, G, I, and V represents the hydrophobic face

significantly vary during the stages of post-harvest ripening, these two DzCAMTAs were not considered as ripening-associated TFs. With the exception of $D z C A M T A 2$ and DzCAMTA4, the expression of all $D z C A M T A$ genes correlated with the transcriptome data. The transcript abundance of all DzCAMTAs was also observed at the pre-harvest stage (Immature1IM1 and Immature 2-IM2) (Additional file 4). Except for DzCAMTA1 and DzCAMTA7, no other $D z C A M T A$ was induced significantly prior to fruit harvesting. Interestingly, in comparison to other DzCAMTAs, DzCAMTA3 and DzCAMTA8 were highly expressed (in comparison to the other six ripening-associated $D z C A M T A s$ ) at the ripe stage. The expression levels of $D z C A M T A 3$ and $D z C A M T A 8$ were also correlated with the transcriptome data (Additional file 5- Fig. A, B, and C). Thus, $D z C A M T A 3$ and DzCAMTA8 were selected for further analysis. The transcript abundance of
$D z C A M T A 3$ and DzCAMTA8 (the two highly expressed putative ripening-associated $D z C A M T A s$ ) was further investigated under ethylene and auxin treatments.

\section{Validation of ripening-associated DzCAMTAs under three ripening treatments and auxin treatment}

Plant CAMTAs were first identified as ethylene-induced CaM-binding proteins $[17,18]$. Nonetheless, the role of ethylene in fruit ripening is well established [4]. Thus, it was interesting to study the effect of ethylene on $D z C A M T A$ s during fruit ripening. The transcript abundance of DzCAMTA3 and DzCAMTA8 (the two highly expressed putative ripening-associated $D z C A M T A$ s) was further investigated under three ripening treatments: natural ripening, ethephon-induced ripening, and 1MCP-delayed ripening. The expression levels of $D z C A M T A 3$ and DzCAMTA8 were significantly increased upon ethephon treatment, but declined 


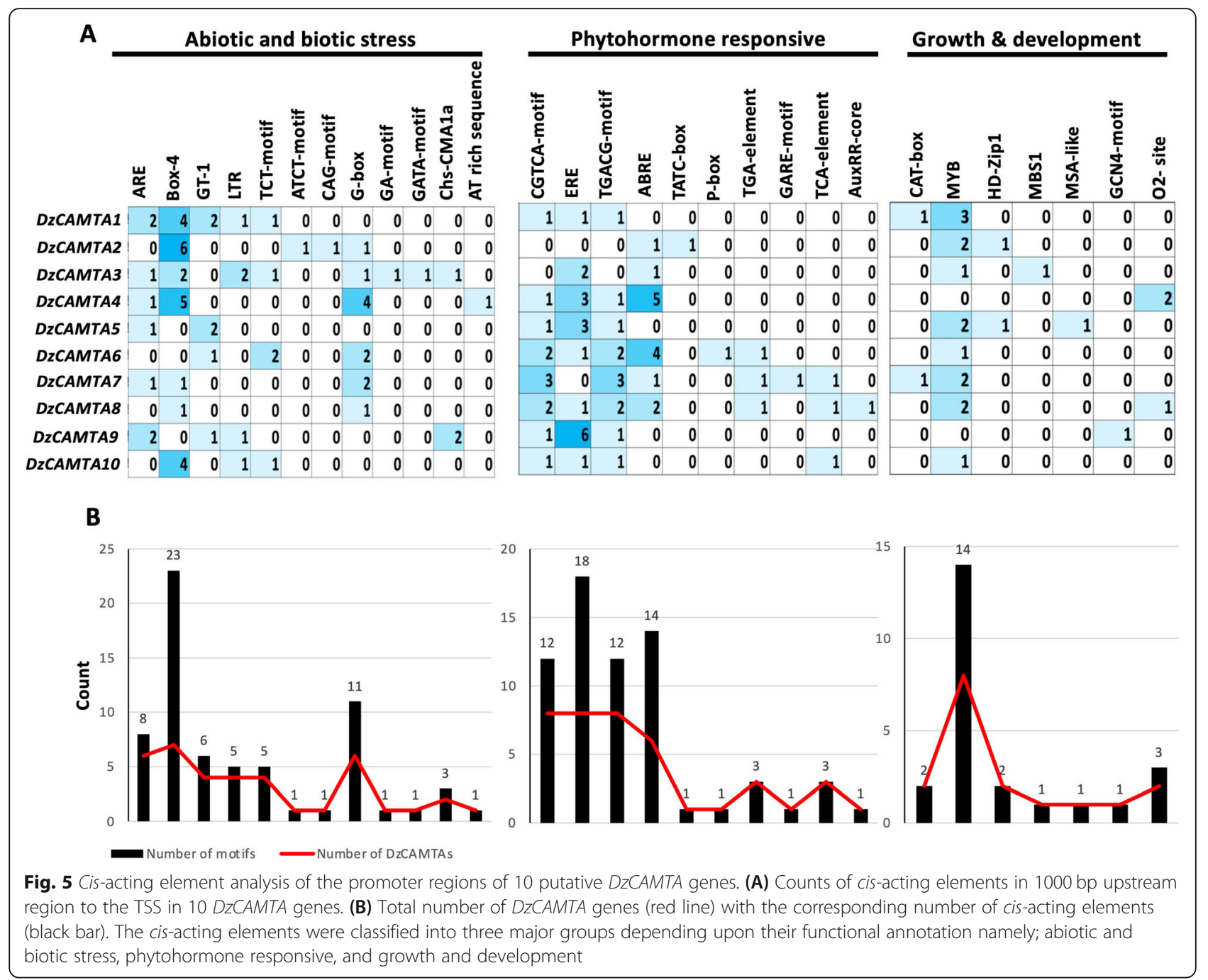

drastically upon 1-MCP treatment in comparison to the natural ripening process (Fig. 7A and $\mathrm{B}$ ). This observation provides strong evidence for the ripening-associated roles of these two DzCAMTAs. Furthermore, during fruit ripening, a cross-wired mesh of interactions between ethylene and auxin exists [67]. Auxin levels had been reported to be significantly altered during the ripening process in durian 'Monthong', thus highlighting the possible ripening-associated role of auxin [68]. This prompted us to further investigate whether there is any correlation between auxin levels and ripening-associated $D z C A M T A$ s (DzCAMTA3 and DzCAMTA8). DzCAMTA3 and $D z C A M T A 8$ expression dramatically declined in a timedependent manner after treatment with $40 \mu \mathrm{M}$ exogenous auxin (Fig. 7C and D).

\section{Co-expression network analysis of DzCAMTA3 and DzCAMTA8 during post- harvest ripening}

Significant RNA-sequencing (RNA-seq) data for the mature and ripe stages of durian 'Monthong' is publicly available.
The variation in the expression of the $10 D z C A M T A s$ at mature and ripe stages were assessed using a box plot (Additional file 5- Fig. A and B). The RNA-seq data also confirmed the high expression of DzCAMTA3 and $D z C A M T A 8$ during post-harvest ripening (Additional file 5- Fig. C). To identify the genes that are co-expressed with $D z C A M T A 3$ and $D z C A M T A 8$ during post-harvest ripening, we analyzed the RNA-seq data of durian 'Monthong' at the mature and ripe stages. The expression values were used in Cytoscape version 3.8.0 to identify the genes that are co-expressed with $D z C A M T A 3$ and DzCAMTA8. A total of 134 DzCAMTA3 positively interacting genes (DzCAMTA3PinG) $(\mathrm{r} \geq 0.95) \quad$ (Fig. 8A and Additional file 1- Table S7) and $126 \mathrm{DzCAMTA3}$ negatively interacting genes $(D z C A M T A 3 N i n G)(r \leq-0.95)$ (Fig. $8 \mathrm{~B}$ and Additional file 1- Table S8) were identified. Similarly, $361 \mathrm{DzCAMTA8}$ positively interacting genes (DzCAMTA8PinG) $(\mathrm{r} \geq 0.95) \quad$ (Fig. $8 \mathrm{~F}$ and Additional file 1- Table S9) and 16 DzCAMTA8 negatively interacting genes $(D z C A M T A 8 N i n G)(\mathrm{r} \leq-0.95)$ (Fig. 


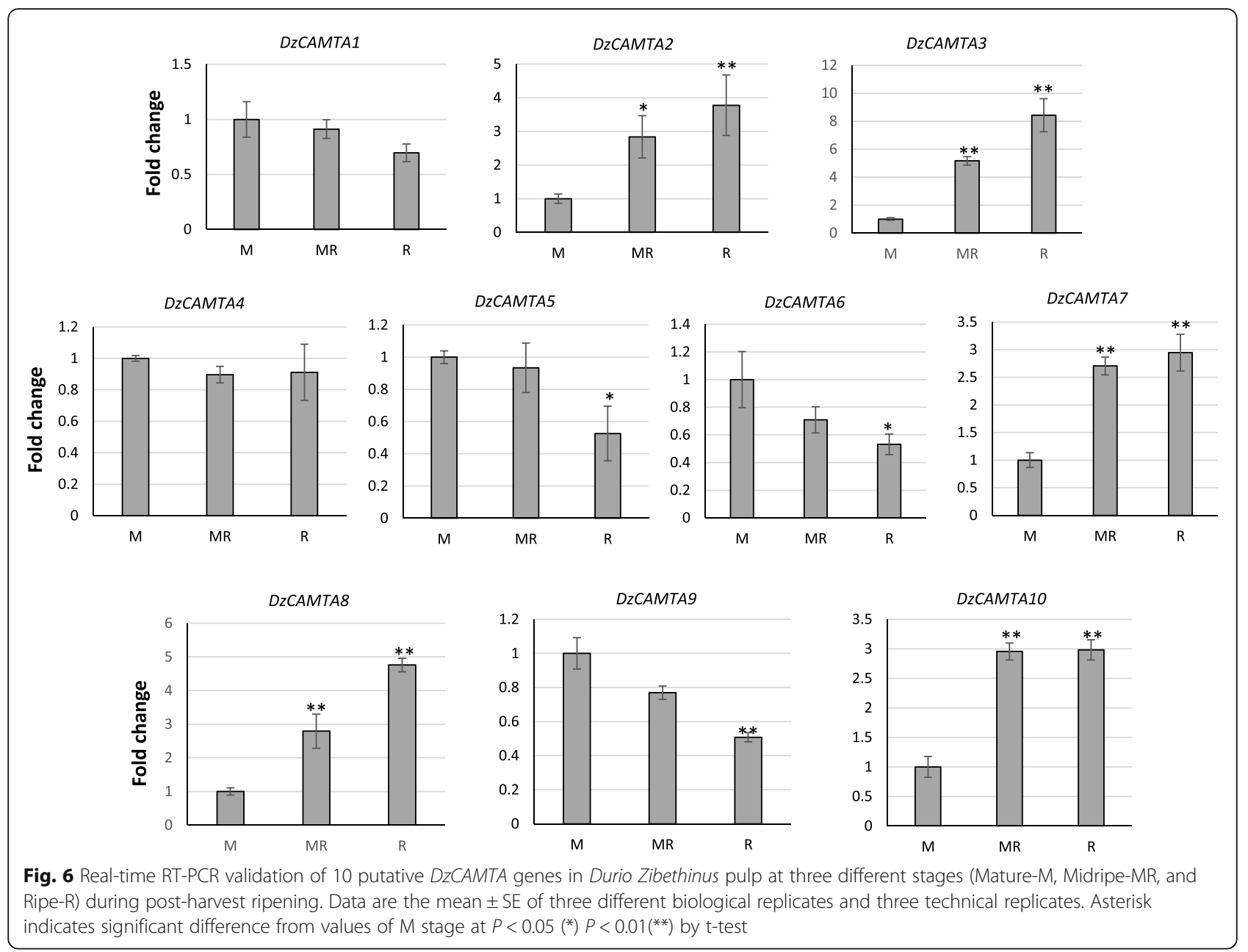

8G and Additional file 1- Table S10) were identified. Next, the cumulative expression of DzCAMTA3PinG, DzCAMTA3NinG, DzCAMTA8PinG, and DzCAMTA8Ning (or so called DzCAMTA3 and $D z C A M T A 8$ interacting genes) were assessed. $D z C A M T A 3 P i n G$ showed significantly higher cumulative expression at the ripe stage than at the mature stage (Fig. 8C), whereas the cumulative expression of DzCAMTA3NinG was lower at the ripe stage (Fig. 8D). In contrast, for DzCAMTA8PinG (Fig. $8 \mathrm{H}$ ) and DzCAMTA8NinG (Fig. 8I), the cumulative expression remained unaltered from the mature to the ripe stage. Furthermore, the promoter regions (1000 bp upstream of the transcription start site) of DzCAMTA3PinG (134), DzCAMTA3NinG (126), DzCAMTA8PinG (361), and DzCAMTA8Ning (16) were scanned to identify the conserved CAMTA recognition motifs (MCGCGB/MCGTGT) (Fig. 8E and $\mathrm{J}$ ). Interestingly, the frequency of occurrence of MCGTGT was higher than that of MCGCGB in all interacting genes. This is because the MCGTGT core motif has an overlapping binding site with the abscisic acid responsive element (ABRE) [41, 69]. It is thus conclusive to say that the presence of CAMTA recognition motifs in the promoter regions of DzCAMTA3PinG, DzCAMTA3NinG, DzCAMTA8PinG, and DzCAMTA8NinG might result in their functional regulation by $D z C A M T A 3$ and DzCAMTA8.

\section{Pathway and gene ontology analysis of genes co- expressed with $D z C A M T A 3$ and DzCAMTA8}

DzCAMTA3PinG, DzCAMTA3NinG, DzCAMTA8PinG, and DzCAMTA8Ning were subjected to MapMan visualization and statistical analysis tool to identify the major pathways regulated by the DzCAMTA3 and $D z C A M T A 8$ co-expressed genes during post-harvest fruit ripening. The MapMan analysis revealed that several DzCAMTA3PinG and DzCAMTA8PinG belonged to TFs representing AP2/ERF, DREB, ARF, SBP, bHLH, MYB, HUA2, WRKY, NAC, HSF, and bHLH families, which have been well documented to be involved in the regulation of fruit ripening processes (Additional file 6Fig. A). Internal plant rhythms, such as the circadian 


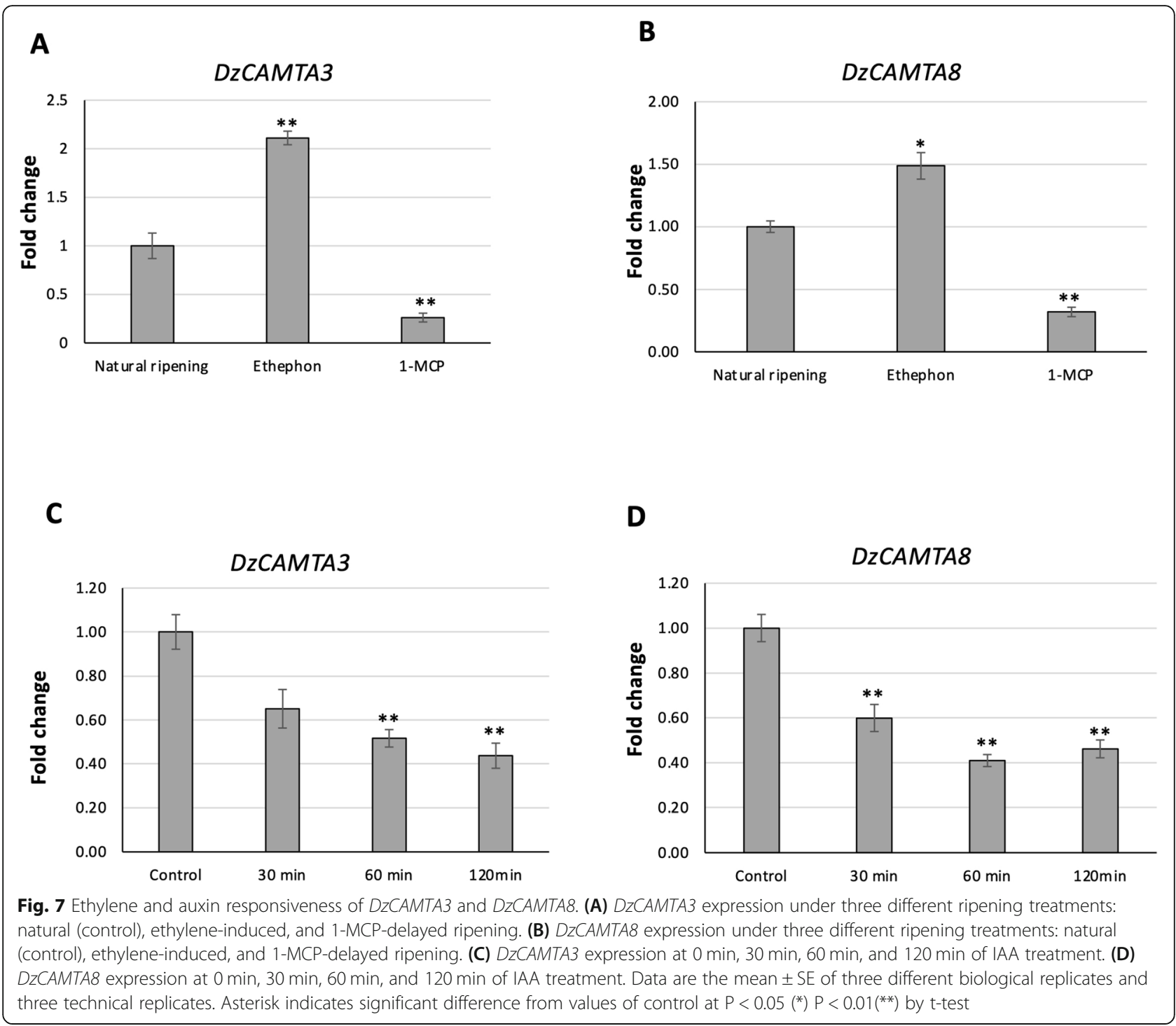

clock, have been shown to affect post-harvest ripening with respect to certain plant metabolites [70]. Several $D z C A M T A 3 P i n G$ and DzCAMTA8PinG were found to regulate circadian clock systems, secondary metabolism, and solute transport (Additional file 6- Fig. B, C, and D). As already discussed, CAMTAs are $\mathrm{Ca}^{2+} / \mathrm{CaM}$-regulated TFs that have long been linked to phytohormones, such as ABA, ethylene, and auxins [14]. Coherently, $D z C A M-$ TA3PinG and DzCAMTA8PinG were linked with positive regulation of ABA, ethylene, and auxins (Additional file 6- Fig. E). Moreover, positive regulation of CDPK, CAMK, and MAPKs were also identified in DzCAMTA3PinG and DzCAMTA8PinG (Additional file 6- Fig. F). Carbohydrate metabolism and cell wall structural changes during post-harvest ripening have long been the subject of study [71, 72]. It was interesting to note that $D z C A M T A 8 P i n G$ were involved in carbohydrate metabolism as well as cell wall and its precursor synthesis
(Additional file 6- Fig. G and H). Additionally, DzCAMTA8Pin $G$ were also involved in external stimulus response (Additional file 6- Fig. I), nutrient uptake (Additional file 6- Fig. J), and photosynthesis (Additional file 6- Fig. K). In contrast, DzCAMTA3NinG and DzCAMTA8NinG were involved in fewer functional processes, including coenzyme metabolism, solute transport by the OPT family, protein modification (TLK, CMGC, and CAMK protein kinase families), and targeting of TF families $(\mathrm{C} 2 \mathrm{H} 2$ and FAR1). Furthermore, GO enrichment analysis was performed to gain insights into the biological processes, molecular functions, and cellular components governed by $D z C A M T A 3$ and $D z C A M T A 8$ co-expressed genes. Taking into account $D z C A M T A 3$ interacting genes, several GO categories related to biotic and abiotic stress, fruit development, seed development, and ripening were significantly affected. This included drought recovery, root hair cell tip growth, pollen tube growth, cell tip growth, pollen 


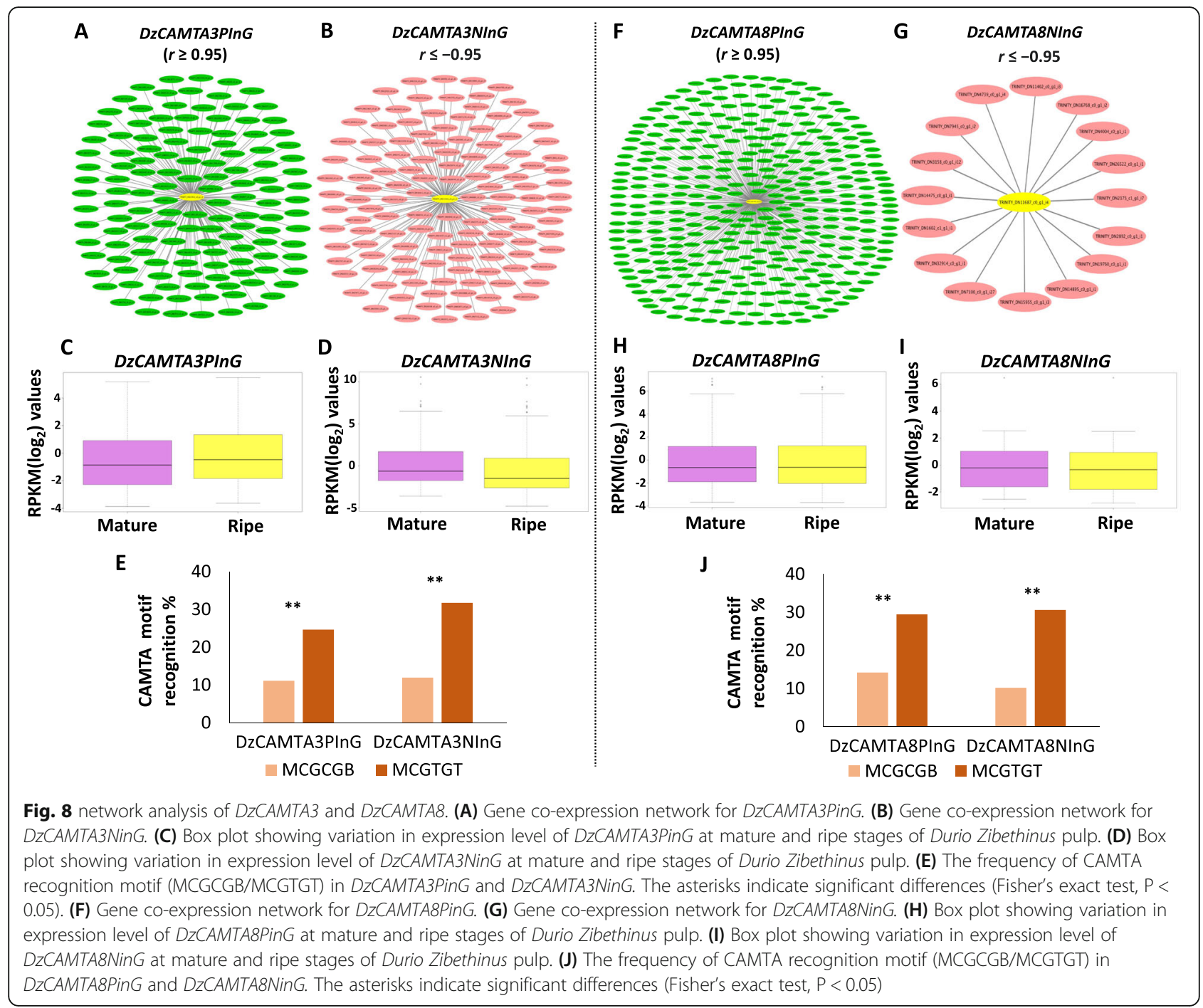

tube development, response to UV, pollination, embryo development ending in seed dormancy, embryo development, seed development, inositol 3-kinase activity, carbohydrate kinase activity, 1-aminocyclopropane-1carboxylate biosynthetic process, and clathrin-coated endocytic vesicle membrane (Additional file 7). The GO categories significantly affected by $D z C A M T A 8$ interacting genes included major biotic stress-related and fruit ripening-related categories such as MAP kinase activity, defense response, response to ethylene, response to salicylic acid, jasmonic acid metabolic process, regulation of ABA signaling pathway, immune system process, response to wounding, response to hydrogen peroxide, and vegetative to reproductive phase transition of meristem. One interesting GO category associated with DzCAMTA8 interacting genes was $\mathrm{CaM}$ binding (molecular function), validating the role of $D z C A M T A 8$ as a CaM-binding TF (Additional file 8). Additionally, the GO categories for the $10 \mathrm{DzCAMTA}$ genes were also assessed (Additional file 9).
As expected, $\mathrm{CaM}$ binding was the highest enriched $\mathrm{GO}$ in the molecular function category for $D z C A M T A s$ (Additional file 10).

\section{Discussion}

The recent availability of the Durio zibethinus draft genome [73] allowed us to perform a comprehensive analysis of the CAMTA gene family in durian with respect to post-harvest ripening. The implication of CAMTA genes in tomato fruit ripening [27] further prompted us to study the role of DzCAMTAs in same direction. We undertook a comprehensive genome-wide characterization and expression analysis to establish the role of DzCAMTA TFs in post-harvest fruit ripening. The present study identified 10 putative $D z C A M T A$ genes in the durian genome (Table 1). Domain organization analysis of DzCAMTAs revealed that except DzCAMTA10 (non-TIG CAMTA), all other DzCAMTAs possessed the conventional CAMTA 
domains (Fig. 1A). Several other plant species, such as Arabidopsis lyrata, Gossypium raimondii, Gossypium hirsutum, Gossypium arboretum, and Capsella rubella lack the TIG domain in their reported CAMTAs [24, 52]. It is hypothesized that during evolution, instead of complete deletion of this domain in CAMTAs, mutation of some vital amino acids probably occurred [52]. Thus, apparently the generation of non-TIG CAMTAs led to the expansion of the CAMTA gene family in durian. All the predicted DzCAMTAs were long proteins comparable to their counterparts in other plant species such as Arabidopsis thaliana [25] and Gossypium [24]. All DzCAMTAs localized to the nucleus with a high reliability index (Table 1), had similar exon-intron organization, and motif structures (Fig. 1C, D and Additional file 1- Table S3). The phylogenetic relationship of DzCAMTAs with other plant species indicated that the major groups contained orthologs from Arabidopsis thaliana, Solanum lycopersicum, Theobroma cacao, and Gossypium hirsutum. This proposes a similar function of DzCAMTAs with other members clustered together. For example, DzCAMTA3 grouped together with Solanum lycopersicum Solyc01g105230 (SISR1L) (Fig. 2). SISR1L is reported during tomato enlargement and ripening [27], which led us to hypothesize the similar function of DzCAMTA3 in durian fruit ripening. Similarly, DzCAMTA8 grouped with AtCAMTA3 (Fig. 2). The involvement of AtCAMTA3 has been shown to regulate biotic stress responses [22, 43, 44]. Coherently, biotic phenomenon also affects fruit ripening and senescence [74].

Hoarding evidences suggest that the expansion of gene families due to gene duplication events is one of the pivotal evolutionary mechanisms contributing to functional diversification and speciation [75]. In concurrence, the localization of paralogous durian CAMTA gene pairs on different scaffolds are indicative of segmental duplication events (Fig. 3). Moreover, the predominance of purifying selection in paralogous durian CAMTA gene pairs implies the selective removal of deleterious alleles. Loss-offunction mutations are possibly obliterated by purifying selection, thus fixing the new duplicate gene and ameliorating functional alleles at both duplicate loci [76]. Interestingly, DzCAMTA1 and DzCAMTA3 (the paralogous gene pair) showed opposite expression profiles during post-harvest ripening (Fig. 6). This substantiates the fact that this paralogous gene pair might have different functions in durian. Furthermore, the duplication time of GhCAMTAs (Gossypium is the closest relative of durian) is 13.04 MYA [24], whereas for DzCAMTAs is 14.64 to 17.95 MYA. This implies that the duplication events in the durian CAMTA families were more ancient than polyploid formation. Probably, these duplications may be responsible for the unique functions of CAMTAs in durian, i.e., post-harvest ripening.
$\mathrm{Ca}^{2+}$ is an important second messenger that mediates responses to external stimuli and hormonal fluctuations via $\mathrm{Ca}^{2+}$ sensors, such as $\mathrm{CaM}[14,77,78]$. The role of $\mathrm{Ca}^{2+}$ is further extended in fruit ripening via the intervention of cross-wired mesh of regulatory networks [27, 77]. Much alike all the other CAMTAs that have been characterized, the 10 DzCAMTAs encoded a type of $\mathrm{Ca}^{2+} / \mathrm{CaM}$-binding TF (Fig. 4). It has been wellestablished that $\mathrm{Ca}^{2+} / \mathrm{CaM}$ binding to CAMTAs is critical for its in-vivo functions $[18,79]$. Therefore, it can be reasonably speculated that one of the mechanisms by which $\mathrm{Ca}^{2+}$ mediates fruit ripening is the formation of a $\mathrm{Ca}^{2+} / \mathrm{CaM}$ complex to activate DzCAMTAs. DzCAMTAs might in-turn regulate the expression of downstream genes involved in fruit ripening process. Thus, DzCAMTAs probably act as co-ordinators for several cross-wired signaling pathways implicated in fruit development and ripening (Fig. 7). Furthermore, the interaction of $\mathrm{CaM}$ with CaMBD occurs in a $\mathrm{Ca}^{2+}$-dependent manner. In contrast, the IQ motif interacts with CaM in a $\mathrm{Ca}^{2+}$-independent manner $[19,20,25,27,52,79]$. Interestingly, all the putative DzCAMTAs possessed a conserved CaMBD adjacent to the IQ motif (Fig. 1A and Fig. 4). This might imply that the interaction of $\mathrm{CaM}$ with DzCAMTAs can occur either in a $\mathrm{Ca}^{2+}$-dependent manner or in a $\mathrm{Ca}^{2+}$-independent manner. Moreover, the invariable presence of certain important development-related (MYB, HD-Zip1, and GCN-4 motif) and phytohormonal-related (ABRE, AuxRR-core, CGTCA, and ERE) cis-acting elements in the promoter region of $D z C A M T A$ s strengthened their involvement in fruit development and ripening (Fig. 5). Conceivably, the occurrence of these motifs in the promoter regions of $D z C A M T A$ s is indicative of the plausible molecular regulation which can be further confirmed through sequencing.

Gene expression analysis was performed to validate the involvement of $D z C A M T A$ s in the post-harvest ripening of durian (Fig. 6, Additional file 4, Additional file 5). Gene expression analysis identified $D z C A M T A 3$ and $D z C A M T A 8$ as the highest expressing $D z C A M T A$ s from mature to ripe stage (post-harvest ripening) (Fig. 6, Additional file 4, Additional file 5). Consequently, $D z C A M T A 3$ and DzCAMTA8 were contemplated for their involvement in post-harvest ripening of durian. As previously discussed, CAMTA TF family was initially identified as an ethylene-induced CaM-binding protein [17]. Ethylene acts as the main phytohormone in regulating the ripening of climacteric fruits. Ethylene regulates fruit ripening via a complex network of interacting signaling pathways and ripening-associated developmental factors. Thus, it was interesting to study the transcript abundance of these putative ripening-associated $D z C A M T A$ s under natural ripening conditions and 
ethylene induced/delayed ripening conditions. The expression levels of $D z C A M T A 3$ and $D z C A M T A 8$ were enhanced under ethephon treatment, while suppressed under 1-MCP treatment (Fig. 7A and B). The effect of ethylene released by ethephon [80] and the ethylene inhibition by 1-MCP $[81,82]$ are well documented. Therefore, the up-regulation of $D z C A M T A 3$ and $D z C A M T A 8$ by ethephon and down-regulation by $1-\mathrm{MCP}$ affirms their involvement in ethylene-induced fruit ripening. Additionally, the transcript abundance of these ethyleneinduced $D z C A M T A$ s increased during post-harvest ripening (Fig. 6, Additional file 4, Additional file 5). Therefore, DzCAMTA3 and DzCAMTA 8 are speculated to function as ethylene-induced transcriptional activators of ripening. Moreover, as mentioned above, in a similar study, the CAMTA TF family member from Solanum lycopersicum (SISR1L) has been reported to be ethyleneinduced (phylogenetically grouped together with $D z C A M T A 3)$ and acts as a transcriptional activator of ripening [27]. This further asserts the observations made in the present study. Similarly, transcriptional regulation of auxin biosynthetic genes has been previously shown to mediate post-harvest ripening of the Monthong cultivar by the joint action of $D z A R F 2 A$ and $D z D o f 2.2$ [68, 83]. In agreement with previous reports, $D z C A M T A 3$ and $D z C A M T A 8$ (Fig. 7C and D) expression dramatically declined in a time-dependent manner upon exogenous auxin treatment. This indicated a positive correlation of ethylene with $D z C A M T A 3$ and $D z C A M T A 8$, while a negative correlation of auxin with DzCAMTA3 and DzCAMTA8. Consequently, DzCAMTA3 and DzCAMTA8 synergistically crosstalk with ethylene during the postharvest ripening of the durian fruit. In contrast, $D z C A M T A 3$ and DzCAMTA8 antagonistically with auxin could mediate the post-harvest ripening of durian fruit.

The co-expression analysis of DzCAMTA3 and $D z C A M T A 8$ suggested that they may be involved in regulating complex gene networks underlying post-harvest ripening of climacteric fruits (Fig. 8A, B, F, G, Additional file 1- Table S7, S8, S9, and S10). DzCAMTA3PinG, DzCAMTA3NinG, $D z C A M T A 8 P i n G$, and $D z C A M T A 8 N i n G$ belonged to UDPsugar pyrophosphorylase [84], heat shock TF [85], E3 ubiquitin ligase [86], GATA zinc finger TF [87], protein kinase [88], NADH dehydrogenase [89], 1-amino-cyclopropane-1carboxylate synthase [90], TIFY [91], cytochrome P450 [92], cyclin dependent kinase [93], ethylene responsive factor [2], homeodomain subfamily [94], WRKY [95], MYB [96], auxin-responsive factor AUX/IAA [83], MAP kinase [97], squamosa promoter binding protein [98], $\mathrm{C} 2 \mathrm{H} 2$ zinc finger protein [99], and AP2/B3 TF [100] protein families, which have been previously reported to play significant roles in fruit ripening. The presence of 1-aminocyclopropane-1-carboxylic acid (ACC) synthase enzyme (catalyzes the synthesis of ACC-precursor of ethylene) in DzCAMTA3NinG was a very interesting observation in the present study with respect to fruit ripening (Additional file 1- Table S8). Our results suggest that CAMTAs can act as both positive and negative regulators of gene expression during fruit ripening. As such, AtCAMTA3 acts as a positive regulator of the CBF2 regulon [42], and also acts as a negative regulator of SA-mediated immune responses $[44,101]$. Thus, the presence of ACC synthase in DzCAMTA3NinG was not surprising. In a similar vein, the presence of ACC oxidase (catalyzes the conversion of ACC to ethylene) in $D z C A M$ TA8PinG was equally interesting (Additional file 1- Table S9). The positive co-expression of DzCAMTA8 with ACC oxidase suggests the involvement of this TF in regulating the intricate signaling network of fruit ripening process. The enrichment of CAMTA recognition motifs (MCGCGB/ MCGTGT) in the promoter sequences of DzCAMTA3-and $D z C A M T A 8$ interacting genes also supports the assertions made in the present study (Fig. $8 \mathrm{E}$ and J). Thus, $D z C A M T A 3$ and $D z C A M T A 8$ via a complex regulatory mesh could regulate fruit ripening through $D z C A M T A 3 P$ inG, DzCAMTA3NinG, DzCAMTA8PinG, and DzCAMTA8NinG. One intriguing observation was the significantly greater frequency of occurrence of the MCGTGT motif than MCGCGB in DzCAMTA3 and DzCAMTA8 interacting genes. A plausible explanation for this observation could be that the MCGTGT core motif has an overlapping binding site with ABRE, which is also recognized by bZIP proteins [41, 69], resulting in a greater frequency. It is also important to mention that plant CAMTAs regulate various stress, phytohormonal, and ROS responses in plants [14]. ROS response, phytohormonal regulation, and redox state are equally imperative for fruit ripening [102-104]. The identification of pathways belonging to these categories in DzCAMTA3PinG, DzCAMTA3NinG, DzCAMTA8PinG, and DzCAMTA8NinG supports the importance of DzCAMTA3 and DzCAMTA8 in fruit ripening (Additional file 6- Fig. A, C and E). Cell wall [105] and carbohydrate metabolism [71] are often linked with fruit ripening, and the regulation of these pathways by DzCAMTA3 and $D z C A M T A 8$ interacting genes further emphasize their involvement in fruit ripening (Additional file 6- Fig. $\mathrm{G}$ and $\mathrm{H}$ ). Finally, the enrichment of certain important GO terms related to ACC biosynthetic process, amino peptidase activity, fructose 2,6-bisphosphate metabolic process, ethylene response, inositol -3 kinase, embryo development, CaM binding, MAPK cascade, etc. in DzCAMTA3 and DzCAMTA8 interacting genes substantiate their role in the fruit ripening process (Additional file 7 and Additional file 8).

\section{Conclusion}

Taken together, our results suggest that $D z C A M T A 3$ and $D z C A M T A 8$ are the key components of the regulatory network underlying post-harvest ripening in durian (Additional file 11). The present study also represents 
another layer of intricate gene regulatory network involved in fruit ripening. The study further emphasizes the fact that the ripening process heavily relies on the interplay between different TFs and hormones. The findings from this study can be applied to the development of molecular markers for producing new durian varieties with prolonged shelf life. Nonetheless, rigorous analysis unraveling the underpinning molecular mechanisms of DzCAMTAs in fruits will provide greater insights into their ripening-associated roles, which remain elusive and a subject for further study.

\section{Methods}

\section{Plant materials and exogenous treatments}

Durian cultivar Monthong (D. zibethinus Murr. 'Monthong') fruits were collected from a commercial orchard at Trat province of Thailand. Durian fruits of almost similar weight $(\sim 3-4 \mathrm{~kg})$ and size were harvested at the immature and mature stages. The immature stage for Monthong was at 85 days after anthesis (DAA) [50, 68, 83], while the mature stage was at 105 DAA $[50,68,83]$. Immature durian fruit samples and some mature durian fruit samples were peeled immediately after harvesting. The remaining mature durian fruit samples were stored at room temperature $\left(\mathrm{RT} \sim 30^{\circ} \mathrm{C}\right)$ until peeling. In this study, durian fruit samples were obtained at five different stages: immature 1, immature 2, mature, mid ripe, and ripe. For mature, mid ripe, and ripe samples, fruits were harvested at the mature stage, kept at RT for one, three, and five days and then peeled, respectively. After peeling, two central pulps from each fruit were collected according to the protocol described in [50]. To ensure that the durian fruit samples were compared at the same ripening stage, the first pulp was collected along with a seed, and fruit firmness was measured using a texture analyzer (TA-XT2i; Stable Micro Systems, Godalming, UK) according to the protocol described in [68]. A puncture test was performed at five random points in each pulp at a test speed of $2 \mathrm{~mm} / \mathrm{s}$ and a test distance of 5 $\mathrm{mm}$ using a $6-\mathrm{mm}$ probe. Midripe pulps had a mean \pm standard deviation (SD) of $3.4 \pm 0.81 \mathrm{~N}$, whereas ripe pulps had a mean \pm SD of $1.55 \pm 0.45 \mathrm{~N}$. After this assessment, the pulp was collected without seeds. It was immediately snap frozen in liquid nitrogen and stored at $-70^{\circ} \mathrm{C}$ for RNA preparation.

Three different ripening treatments (natural ripening, ethephon-induced, and 1-methylcyclopropene (1-MCP)delayed ripening) were compared to study the effect of ethylene on $D z C A M T A 3$ and $D z C A M T A 8$ during postharvest ripening. Ethephon (48\% 2-chloroethylphosphonic acid; Alpha Agro Tech Co., Ltd., Thailand) solution was applied to the upper area of each fruit stalk (mature durian samples). For treating the mature durian samples with 1 -MCP, each fruit was placed in a $20 \mathrm{~L}$ closed chamber.
To this chamber one tablet of 1-MCP (0.19\% 1-MCP tablet; BioLene Co., Ltd., China) was placed in a beaker. Five milliliters water was added to the beaker containing 1$\mathrm{MCP}$ tablet to produce gaseous 1-MCP. The chamber was immediately closed at RT for $12 \mathrm{~h}$. Control samples were treated in the same manner at the same temperature and for the same period of time without 1-MCP. After $12 \mathrm{~h}$, the control and 1-MCP-treated samples were kept at RT for ripening and collected the same day when ethephon treated durian samples were collected. The samples were then peeled and processed as described previously. Three independent biological replicates of each sample type were used in this study. Each biological replicate represented one durian fruit, which was harvested from a separate tree.

For exogenous auxin application, young leaves of $D$. zibethinus Murr. 'Monthong' were soaked in $40 \mu \mathrm{M}$ of indole-3-acetic acid (IAA) (Duchefa Biochemie, the Netherlands) for 30, 60, and $120 \mathrm{~min}$. For the control samples, the leaves were soaked in distilled water without IAA. Immediately after the completion of the treatment, the leaves were snap frozen in liquid nitrogen and stored at $-70^{\circ} \mathrm{C}$ for RNA preparation.

\section{Identification of CAMTA gene family in Durio zibethinus}

The whole-genome protein sequence of Durio zibethinus was downloaded from the National Center for Biotechnology Information (NCBI) (https://www.ncbi.nlm.nih. gov/). A total of 1343 CAMTA protein domain sequences from 166 plant species were downloaded from the Plant Transcription Factor Database (http:// planttfdb.gao-lab.org/index.php) [106]. These two sets of data were used to construct a Hidden Markov Model (HMM) profile. Subsequently, the HMM profile of the conserved CAMTA domains (CG-1, CaMBD, TIG domain, ankyrin repeats, and IQ) was utilized as a query in the HMMER (V3.0) software to identify the putative Durio zibethinus CAMTAs. The redundant and partial sequences were removed and the $D z$ CAMTAs were named numerically.

\section{Domain organization, gene structure and conserved motif analysis of DzCAMTAs}

All $D z$ CAMTAs were subjected to the Pfam database (http://pfam.xfam.org/), NCBI Conserved Domain Database (https://www.ncbi.nlm.nih.gov/cdd/), and InterPro database (https://www.ebi.ac.uk/interpro/search/ sequence/) to confirm the presence of conserved CAMTA domains. Nuclear localization signal was queried using the Motif Scan (http://myhits.isb-sib.ch/cgibin/motif_scan) and NLS Mapper (http://nls-mapper.iab. keio.ac.jp/cgi-bin/NLS_Mapper_form.cgi). CaMBD was mapped using the Calmodulin Target Database (http:// calcium.uhnres.utoronto.ca/ctdb/ctdb/home.html). 
Finally, a schematic representation of the $D z$ CAMTAs functional domains was constructed using Illustrator for Biological Sequences software (http://ibs.biocuckoo.org/) [107]. The gene structures of $D z C A M T A$ s were analyzed by querying $D z C A M T A$ coding sequences with their corresponding genomic sequences on the Gene Structure Display Server (GSDS) (http://gsds.cbi.pku.edu.cn/) [108]. Conserved protein motifs were determined using the Multiple Em for Motif Elicitation (MEME) tool (https://meme-suite.org/meme/tools/meme) [109] with the following parameters: width from " 6 to 300 ", "zero" or "one" per sequence, and maximum number of motifs to search " 20 ". The motifs were annotated using the InterproScan software.

\section{Computation of physicochemical properties and subcellular localization of DzCAMTAs}

The physicochemical properties of the DzCAMTA proteins were computed using ProtParam (http://web. expasy.org/protparam/). The subcellular localization of DzCAMTA proteins was determined by CELLO v.2.5, (http://cello.life.nctu.edu.tw/).

\section{Multiple sequence alignment (MSA) and phylogenetic tree construction of DzCAMTA proteins}

CAMTA protein sequences from four different plant species were downloaded from the Plant Transcription Factor Database (http://planttfdb.gao-lab.org/index.php) [106]. MSA was performed using the Clustal Omega program (https://www.ebi.ac.uk/Tools/msa/clustalo/) with default parameters. MEGA X software [110] was used to construct a maximum likelihood (ML) phylogenetic tree. The parameters employed included pairwise gap deletion, the JTT model, and 1000 iterations of bootstrap values.

\section{Scaffold gene physical position and gene duplication analysis}

The physical locations of all 10 DzCAMTAs were obtained using a BLASTN search. The DzCAMTA genes were mapped onto the related scaffolds using Mapinspect software. The paralogous durian CAMTA gene pairs were identified by reciprocal blast analysis with an e-value $<10^{-5}$. The parameters used were query coverage $>70 \%$ of the longer sequence and percentage identity $>70 \%$ [111]. Ka/Ks analysis was performed using PAL2NAL [112]. The Ks values attained for each gene pair were converted into divergence time in million years ago (MYA), assuming a rate of $6.1 \times 10^{-9}$ substitutions per site per year for eudicots $[75,113]$. The divergence time (T) was estimated as $\mathrm{T}=\mathrm{Ks} / 2 \lambda(\lambda=6.1 \times$ $10^{-9}$ ) [114]. Further, a $\mathrm{Ka} / \mathrm{Ks}$ ratio $<1$ indicates negative (purifying selection), $>1$ indicates positive selection, and $=1$ indicates neutral selection [115].
RNA-seq analysis, generation of heat map and box plot Illumina sequencing reads from RNA-seq of durian (Monthong cultivar) were retrieved from the public repository Sequence Read Archive (SRA) at NCBI (project number: PRJNA683229) [116]. The method in [68] was used to obtain the de-novo assembled transcriptome. The input reads were aligned to the de-novo assembled transcriptome using the abundance estimation tool of the Trinity package to generate raw counts for each contig. Raw read counts were merged into a single read count matrix. The single read count matrix was normalized to obtain a trimmed mean of the M-values (TMM) normalized matrix. Normalized total read counts were used to generate a heatmap. The heatmap was drawn using MetaboAnalyst 5.0 [117], an R-based public database. The parameters used for heatmap generation were as follows: normalization by a pooled sample from the group, $\log _{2}$ transformed, and autoscaled. Similarly, box plots were drawn using the ggplot2 (https://cran.rproject.org/web/packages/ggplot2/) package in $\mathrm{R}$ version 4.0.3 GUI 1.73.

\section{Co-expression network and promoter analysis of DZCAMTA3 and DzCAMTA8 interacting genes}

Expression values were used to generate a gene coexpression network. The "Expression Correlation Networks" (http://apps.cytoscape.org/apps/ expressioncorrelation) plugin of Cytoscape version 3.8.0. was used to calculate positive Pearson correlation (default $r \geq 0.95$ ) and "anti-correlation" or negative Pearson correlation (default $r \leq-0.95$ ) in the interacting members of a network. Network visualization was performed by applying a forcedirected layout in Cytoscape. The nodes (circles) represent genes and edges (straight lines) represent significant interactions between the expression levels of genes from the mature to the ripe stage (gene correlation network). The promoter sequences with the required length of $1000 \mathrm{bp}$ for $D z C A M T A 3$ and $D z C A M T A 8$ interacting genes were fetched by running a shell script on a multi-fasta file with genomic sequences and a GFF file for the multi-fasta file available at NCBI. The other dependencies (required packages) included Samtools (http://www.htslib.org/) and bedtools (https://bedtools.readthedocs.io/en/ latest/). Further, another in-house python script was run to identify the MCGCGB and MCGTGT motifs in the promoter regions (complement and reverse complement) of DzCAMTA3 and DzCAMTA8 interacting genes.

\section{Promoter cis-acting element analysis of DzCAMTAs}

DNA sequences $1000 \mathrm{bp}$ upstream of the transcription start site for DzCAMTAs were retrieved from NCBI. 
These sequences were queried using PlantCARE [53] (http://bioinformatics.psb.ugent.be/webtools/plantcare/ $\mathrm{html} /$ ) and PLACE database (https://www.dna.affrc.go. jp/PLACE/?action=newplace) [54] for the identification of cis-acting elements.

\section{Pathway and gene ontology analysis of DzCAMTA3 and DzCAMTA8 interacting genes}

Pathway analysis of DzCAMTA3PinG, DzCAMTA3NinG, $D z C A M T A 8 P i n G$, and DzCAMTA8NinG was performed using MapMan software (http://www.gabipd.org/projects/ MapMan/) [118]. Multiple correction tests (Benjamini Hochberg) with FDR $<0.05$, were used to fetch significant functional categories or metabolic pathways. Furthermore, the Blast2GO feature of OmicsBox (https://www.blast2go. $\mathrm{com} /$ ) was utilized to identify significant Gene Ontology (GO) terms with respect to three aspects: biological processes, molecular functions, and cellular components.

\section{RNA isolation and real-time quantitative RT-PCR}

Total RNA was isolated from durian pulp. The isolated RNA was purified using PureLink Plant RNA Reagent (Thermo Fisher Scientific, MA, US) according to the manufacturer's protocol. Genomic DNA contamination was removed by treating the purified RNA with DNase I (Thermo Fisher Scientific, MA, US). RNA integrity was assessed by agarose gel electrophoresis and an Eppendorf BioPhotometer D30 with $\mathrm{A}_{260} / \mathrm{A}_{280}$ (ratio between 1.8 to 2.0) and $A_{260} / A_{230}$ (ratios between 2.0 to 2.2). One microgram of DNase I-treated RNA was used to synthesize first-strand cDNA. cDNA was prepared using the RevertAid First Strand cDNA Synthesis Kit (Thermo Fisher Scientific, MA, US) according to the manufacturer's instructions. The elongation factor 1 alpha (EF$1 \alpha)$ gene was used as an internal control. The qRT-PCR reaction was performed in a $20-\mu \mathrm{L}$ reaction volume by adding $5 \mathrm{pmol}$ of gene-specific primers, $1 \mu \mathrm{L}$ cDNA of each sample to $2 \mathrm{X}$ Luna Universal qPCR Master Mix (New England Biolabs, MA, USA). PCR was carried out on a CFX95 Real-time System (Bio-Rad Laboratories Inc., CA, USA) with the following program: initial denaturation at $95^{\circ} \mathrm{C}$ for $1 \mathrm{~min}$, followed by $40-45$ cycles of denaturation at $95^{\circ} \mathrm{C}$ for $15 \mathrm{~s}$, and annealing/extension at $60^{\circ} \mathrm{C}$ for $30 \mathrm{~s}$. Melt curve analysis to examine the specificity of primers was performed from $60^{\circ} \mathrm{C}$ to $95^{\circ} \mathrm{C}$ in increments of $0.5^{\circ} \mathrm{C}$. Transcript abundance was calculated as a comparative fold change following the $2^{-\Delta \Delta \mathrm{ct}}$ quantitative method [119]. Three independent biological replicates and three technical replicates were used for the statistical analysis.

\section{Abbreviations}

CAMTA: Calmodulin binding transcription activator; DzCAMTA3PinG: DzCAMTA3 positively interacting genes; DzCAMTA3NinG: DzCAMTA3 negatively interacting genes;
DzCAMTA8PinG: DzCAMTA8 positively interacting genes; DzCAMTA8NinG: DzCAMTA8 negatively interacting genes

\section{Supplementary Information}

The online version contains supplementary material available at https://doi. org/10.1186/s12864-021-08022-1.

Additional file 1: Table S1. All identified durian CAMTAs. Table S2. Protein sequence of DzCAMTAs. Table S3. List of 20 conserved protein motif sequences and their annotation in DzCAMTAs. Table S4. List of CAMTA genes from five species used for the phylogenetic analysis of DzCAMTAs. Table S5. The $\mathrm{Ka} / \mathrm{Ks}$ ratios and date of duplication for duplicate CAMTA genes in Durio zibethinus. Table S6. List of predicted cis-acting elements in the promoter regions of DzCAMTAs. Table S7. List of positively interacting genes (Pearson correlation coefficient $r \geq 0.95$ ) with DzCAMTA3. Table S8. List of negatively interacting genes (Pearson correlation coefficient $r \leq-0.95$ ) with DzCAMTA3. Table S9. List of positively interacting genes (Pearson correlation coefficient $r \geq 0.95$ ) with DzCAMTA8. Table S10. List of negatively interacting genes (Pearson correlation coefficient $r \leq-0.95)$ with DzCAMTA8.

Additional file 2. Alignment of 10 putative DzCAMTAs proteins with 6 Arabidopsis CAMT As. The conserved domains associated with CAMTA proteins (CG-1, NLS, TIG domain, ankyrin repeat, IQ motif, and CaMBD) are marked below the alignment by orange line.

Additional file 3. Twenty enriched motifs in 10 putative DzCAMTAs. Additional file 4. Real time validation of 10 putative DzCAMTA genes in Durio Zibethinus pulp at five different stages (Immature1-IM1, Immature2IM2, Mature-M, Mid-ripe-MR, and Ripe-R) during post-harvest ripening. Data are the mean \pm SE of three different biological replicates and three technical replicates. Asterisk indicates significant difference from values of $\mathrm{M}$ at $P<0.05\left(^{*}\right) P<0.01\left(^{* *}\right)$ by t-test.

Additional file 5. Expression profile of DzCAMTAs during post-harvest ripening. Variation in expression of 10 DzCAMTAs at (A) mature stage and (B) ripe stage of Durio Zibethinus was visualized by box plot. Each DzCAMTA is represented with a different color. The central line for each box plot indicates the median value. The expression values correspond to the RPKM. (C) Expression profiles of 10 DzCAMTAs in mature and ripe stages of Durio Zibethinus pulp. Data was sum normalized, log transformed, and auto scaled.

Additional file 6. Functional and GO annotation of DzCAMTA3 and DzCAMTA8 interacting genes. MapMan based functional classification of DzCAMTA3PinG, DzCAMTA3NinG, DzCAMTA8PinG, and DzCAMTA8NinG. (A) RNA biosynthesis transcriptional regulation. (B) Multi-process regulation. (C) Coenzyme metabolism, secondary metabolism and redox homeostasis. (D) Solute transport. (E) Phytohormone action. (F) Protein modification. (G) Carbohydrate metabolism. (H)Cell wall organization. (I) External stimuli response. (J) Nutrient uptake. (K) Photosynthesis. The scale represents expression values in $\log _{2}$.

Additional file 7. GO annotation of DzCAMTA3PinG, and DzCAMTA3NinG with respect to biological processes, molecular functions and cellular components, respectively.

Additional file 8. GO annotation of DzCAMTA8PinG, and DzCAMTA8NinG with respect to biological processes, molecular functions and cellular components, respectively.

Additional file 9. GO functional annotation for the 10 DzCAMTAs. The different colors represent the proportions of various $\mathrm{GO}$ terms.

Additional file 10. GO annotation of 10 DzCAMTAs with respect to biological processes, molecular functions and cellular components, respectively.

Additional file 11. Schematic representation of the role of ripening associated DzCAMTA3, and DzCAMTA8 in the regulatory network of durian fruit ripening.

\section{Acknowledgements}

$\mathrm{Zl}$ is thankful to Dr. Teerapong Buaboocha for providing the facilities and infrastructure. $\mathrm{Zl}$ is also thankful to Danyal, Kamonwan Weerawanich, Kittiya 
Tantisuwanichkul, Nattana Thongsima, and Navarit Jitsamai for their valuable inputs during the research.

\section{Authors' contributions}

ZI conducted the experiments, analyzed the data, and drafted the manuscript, MSI helped in drawing the figures, writing the manuscript and downstream analysis, LS performed the transcriptome analysis, GK helped in the transcriptome analysis, SS reviewed the manuscript, and TB conceptualized the idea and reviewed the manuscript. The authors read and approved the final manuscript.

\section{Funding}

This work was funded by research grants from the Second Century Fund (C2F Post-Doc Fellowship). The fellowship for Zl was supported by C2F, Chulalongkorn University. This research was also funded by the National Research Council of Thailand [grant number NRCT5-RSA63001-15].

\section{Availability of data and materials}

The Illumina RNA-seq sequencing reads used in the current study are available in the public repository Sequence Read Archive (SRA) at NCBI, and the project number is PRJNA683229 (https://www.ncbi.n/m.nih.gov/Traces/study/ ?acc $=$ PRJNA683229).

\section{Declarations}

\section{Ethics approval and consent to participate}

Plant material was obtained from commercial orchards in Trat, Thailand $\left(12^{\circ} 09^{\prime} 56.6^{\prime \prime} \mathrm{N} 102^{\circ} 41^{\prime} 13.1^{\prime \prime} \mathrm{E}\right)$. No special permission was necessary to collect such samples. The collection of plant materials complies with national and international guidelines.

\section{Consent for publication}

Not applicable.

\section{Competing interests}

The authors declare no conflict of interest.

\section{Author details}

'Molecular Crop Research Unit, Department of Biochemistry, Chulalongkorn University, Bangkok, Thailand. ${ }^{2}$ Amity Institute of Biotechnology, Amity University, Lucknow Campus, Lucknow, Uttar Pradesh, India. ${ }^{3}$ Omics Sciences and Bioinformatics Center, Faculty of Science, Chulalongkorn University, Bangkok, Thailand.

Received: 4 May 2021 Accepted: 13 September 2021

\section{Published online: 14 October 2021}

\section{References}

1. Alexander L, Grierson D. Ethylene biosynthesis and action in tomato: a model for climacteric fruit ripening. J Exp Bot. 2002;53(377):2039-55. https:// doi.org/10.1093/jxb/erf072.

2. Gao J, Zhang Y, Li Z, Liu M. Role of ethylene response factors (ERFs) in fruit ripening. Food Quality and Safety. 2020;4(1):15-20. https://doi.org/10.1093/ fasafe/fyz042.

3. Li S, Chen K, Grierson D. A critical evaluation of the role of ethylene and MADS transcription factors in the network controlling fleshy fruit ripening. New Phytol. 2019;221(4):1724-41. https://doi.org/10.1111/nph.15545.

4. Liu M, Pirrello J, Chervin C, Roustan J-P, Bouzayen M. Ethylene control of fruit ripening: revisiting the complex network of transcriptional regulation. Plant Physiol. 2015;169(4):2380-90. https://doi.org/10.1104/pp.15.01361.

5. Giovannoni JJ. Genetic regulation of fruit development and ripening. Plant Cell. 2004;16(suppl 1):S170-80. https://doi.org/10.1105/tpc.019158.

6. Matas AJ, Gapper NE, Chung M-Y, Giovannoni JJ, Rose JK. Biology and genetic engineering of fruit maturation for enhanced quality and shelf-life. Curr Opin Biotechnol. 2009;20(2):197-203. https://doi.org/10.1016/j.copbio.2 009.02.015.

7. Gao Y, Zhu N, Zhu X, Wu M, Jiang C-Z, Grierson D, et al. Diversity and redundancy of the ripening regulatory networks revealed by the fruitENCODE and the new CRISPR/Cas9 CNR and NOR mutants. Horticulture Research. 2019;6(1):1-10. https://doi.org/10.1038/s41438-019-0122-x.
8. Osorio S, Scossa F, Fernie A. Molecular regulation of fruit ripening. Front Plant Sci. 2013:4:198. https://doi.org/10.3389/fpls.2013.00198.

9. Dwivany F, Esyanti RR, Robertlee J, Paramaputra IC, Permatadewi RK, Tambun DH, Handayani RU, Pratiwi AS, Zaskia H: Environment effect on fruit ripening related gene to develop a new post harvest technology. In: AIP Conference Proceedings: 2014: American Institute of Physics; 2014: 285-287.

10. Chéour F, Souiden Y. Calcium delays the postharvest ripening and related membrane-lipid changes of tomato. J Nutr Food Sci. 2015;5(5):1. https://doi. org/10.4172/2155-9600.1000393.

11. Elbagoury MM, Turoop L, Runo S, Sila DN. Regulatory influences of methyl jasmonate and calcium chloride on chilling injury of banana fruit during cold storage and ripening. Food Sci Nutr. 2020;9(2):929-42.

12. Ranty B, Aldon D, Cotelle V, Galaud J-P, Thuleau P, Mazars C. Calcium sensors as key hubs in plant responses to biotic and abiotic stresses. Front Plant Sci. 2016:7:327. https://doi.org/10.3389/fpls.2016.00327.

13. Xu W, Huang W. Calcium-dependent protein kinases in phytohormone signaling pathways. Int J Mol Sci. 2017;18(11):2436. https://doi.org/10.3390/ ijms18112436.

14. Iqbal Z, labal MS, Singh SP, Buaboocha T. Ca2+/calmodulin complex triggers CAMTA transcriptional machinery under stress in plants: signaling cascade and molecular regulation. Front Plant Sci. 2020;11:598327. https:// doi.org/10.3389/fpls.2020.598327.

15. Kudla J, Becker D, Grill E, Hedrich R, Hippler M, Kummer U, et al. Advances and current challenges in calcium signaling. New Phytol. 2018;218(2):41431. https://doi.org/10.1111/nph.14966.

16. Mohanta TK, Kumar P, Bae H. Genomics and evolutionary aspect of calcium signaling event in calmodulin and calmodulin-like proteins in plants. BMC Plant Biol. 2017;17(1):1-19. https://doi.org/10.1186/s12870-017-0989-3.

17. Reddy A, Reddy VS, Golovkin M. A calmodulin binding protein from Arabidopsis is induced by ethylene and contains a DNA-binding motif. Biochem Biophys Res Commun. 2000;279(3):762-9. https://doi.org/10.1006/ bbrc.2000.4032.

18. Yang T, Poovaiah B. An early ethylene up-regulated gene encoding a calmodulin-binding protein involved in plant senescence and death. J Biol Chem. 2000;275(49):38467-73. https://doi.org/10.1074/jbc.M003566200.

19. Finkler A, Ashery-Padan R, Fromm H. CAMTAs: calmodulin-binding transcription activators from plants to human. FEBS Lett. 2007:581(21):38938. https://doi.org/10.1016/j.febslet.2007.07.051

20. Yang T, Poovaiah B. A calmodulin-binding/CGCG box DNA-binding protein family involved in multiple signaling pathways in plants. J Biol Chem. 2002; 277(47):45049-58. https://doi.org/10.1074/jbc.M207941200.

21. Chung J-S, Koo SC, Jin BJ, Baek D, Yeom S-I, Chun HJ, et al. Rice CaMbinding transcription factor (OsCBT) mediates defense signaling via transcriptional reprogramming. Plant Biotechnology Reports. 2020;14(3):113. https://doi.org/10.1007/s11816-020-00603-y.

22. Kim Y, Gilmour SJ, Chao L, Park S, Thomashow MF. Arabidopsis CAMTA transcription factors regulate pipecolic acid biosynthesis and priming of immunity genes. Mol Plant. 2020;13(1):157-68. https://doi.org/10.1016/j. molp.2019.11.001.

23. Shkolnik D, Finkler A, Pasmanik-Chor M, Fromm H. CALMODULIN-BINDING TRANSCRIPTION ACTVATOR 6: a key regulator of $\mathrm{Na}$ + homeostasis during germination. Plant Physiol. 2019;180(2):1101-18. https://doi.org/10.1104/pp.19.00119.

24. Pant P, labal Z, Pandey BK, Sawant SV. Genome-wide comparative and evolutionary analysis of calmodulin-binding transcription activator (CAMTA) family in Gossypium species. Sci Rep. 2018;8(1):1-17. https://doi.org/10.1038/ s41598-018-23846-w.

25. Bouché N, Scharlat A, Snedden W, Bouchez D, Fromm H. A novel family of calmodulin-binding transcription activators in multicellular organisms. J Biol Chem. 2002;277(24):21851-61. https://doi.org/10.1074/ jbc.M200268200.

26. Choi MS, Kim MC, Yoo JH, Moon BC, Koo SC, Park BO, et al. Isolation of a calmodulin-binding transcription factor from rice (Oryza sativa L.). J Biol Chem. 2005;280(49):40820-31. https://doi.org/10.1074/jbc.M504616200.

27. Yang $T$, Peng $H$, Whitaker BD, Conway WS. Characterization of a calcium/ calmodulin-regulated SR/CAMTA gene family during tomato fruit development and ripening. BMC Plant Biol. 2012;12(1):1-13. https://doi. org/10.1186/1471-2229-12-19.

28. Shangguan $L$, Wang $X$, Leng $X$, Liu $D$, Ren $G$, Tao $R$, et al. Identification and bioinformatic analysis of signal responsive/calmodulin-binding transcription activators gene models in Vitis vinifera. Mol Biol Rep. 2014;41(5):2937-49. https://doi.org/10.1007/s11033-014-3150-5. 
29. Yang $Y$, Sun T, Xu L, Pi E, Wang S, Wang H, et al. Genome-wide identification of CAMTA gene family members in Medicago truncatula and their expression during root nodule symbiosis and hormone treatments. Front Plant Sci. 2015;6:459. https://doi.org/10.3389/fpls.2015.00459.

30. Leng X, Han J, Wang X, Zhao M, Sun X, Wang C, et al. Characterization of a Calmodulin-binding Transcription Factor from Strawberry (Fragariax ananassa). plant genome. 2015;8(2):plantgenome2014.2008.0039.

31. Wang G, Zeng H, Hu X, Zhu Y, Chen Y, Shen C, et al. Identification and expression analyses of calmodulin-binding transcription activator genes in soybean. Plant Soil. 2015;386(1):205-21. https://doi.org/10.1007/s11104014-2267-6.

32. Yue R, Lu C, Sun T, Peng T, Han X, Qi J, et al. Identification and expression profiling analysis of calmodulin-binding transcription activator genes in maize (Zea mays L.) under abiotic and biotic stresses. Frontiers in plant science. 2015;6:576.

33. Rahman H, Xu Y-P, Zhang X-R, Cai X-Z. Brassica napus genome possesses extraordinary high number of CAMTA genes and CAMTA3 contributes to PAMP triggered immunity and resistance to Sclerotinia sclerotiorum. Front Plant Sci. 2016;7:581. https://doi.org/10.3389/fpls.2016.00581.

34. Wei M, Xu X, Li C. Identification and expression of CAMTA genes in Populus trichocarpa under biotic and abiotic stress. Sci Rep. 2017;7(1):1-10. https:// doi.org/10.1038/s41598-017-18219-8.

35. Kakar KU, Nawaz Z, Cui Z, Cao P, Jin J, Shu Q, et al. Evolutionary and expression analysis of CAMTA gene family in Nicotiana tabacum yielded insights into their origin, expansion and stress responses. Sci Rep. 2018;8(1): 1-14. https://doi.org/10.1038/s41598-018-28148-9.

36. Zhang J, Pan X, Ge T, Yi S, Lv Q, Zheng Y, et al. Genome-wide identification of citrus CAMTA genes and their expression analysis under stress and hormone treatments. J Hortic Sci Biotechnol. 2019;94(3):331-40. https://doi. org/10.1080/14620316.2018.1504631.

37. Meer L, Mumtaz S, Labbo AM, Khan MJ, Sadiq I. Genome-wide identification and expression analysis of calmodulin-binding transcription activator genes in banana under drought stress. Sci Hortic. 2019;244:10-4. https://doi.org/1 0.1016/j.scienta.2018.09.022.

38. Büyük I, IIlhan E, Şener D, Özsoy AU, Aras S. Genome-wide identification of CAMTA gene family members in Phaseolus vulgaris $L$. and their expression profiling during salt stress. Mol Biol Rep. 2019;46(3):2721-32. https://doi. org/10.1007/s11033-019-04716-8.

39. Ali E, Raza MA, Cai M, Hussain N, Shahzad AN, Hussain M, et al. Calmodulinbinding transcription activator (CAMTA) genes family: genome-wide survey and phylogenetic analysis in flax (Linum usitatissimum). PLoS One. 2020; 15(7):e0236454. https://doi.org/10.1371/journal.pone.0236454.

40. Kim Y, Park S, Gilmour SJ, Thomashow MF. Roles of CAMTA transcription factors and salicylic acid in configuring the low-temperature transcriptome and freezing tolerance of a rabidopsis. Plant J. 2013;75(3):364-76. https://doi. org/10.1111/tpj.12205.

41. Pandey N, Ranjan A, Pant P, Tripathi RK, Ateek F, Pandey HP, et al. CAMTA 1 regulates drought responses in Arabidopsis thaliana. BMC Genomics. 2013; 14(1):1-23. https://doi.org/10.1186/1471-2164-14-216

42. Doherty CI, Van Buskirk HA, Myers SJ, Thomashow MF. Roles for Arabidopsis CAMTA transcription factors in cold-regulated gene expression and freezing tolerance. Plant Cell. 2009;21(3):972-84. https:/doi.org/10.1105/tpc.108.063958.

43. Jacob F, Kracher B, Mine A, Seyfferth C, Blanvillain-Baufumé S, Parker JE, et al. A dominant-interfering camta3 mutation compromises primary transcriptional outputs mediated by both cell surface and intracellular immune receptors in Arabidopsis thaliana. New Phytol. 2018;217(4):1667-80. https://doi.org/10.1111/nph.14943.

44. Kim YS, An C, Park S, Gilmour SJ, Wang L, Renna L, et al. CAMTA-mediated regulation of salicylic acid immunity pathway genes in Arabidopsis exposed to low temperature and pathogen infection. Plant Cell. 2017;29(10):2465-77. https://doi.org/10.1105/tpc.16.00865.

45. Galon Y, Aloni R, Nachmias D, Snir O, Feldmesser E, Scrase-Field S, et al. Calmodulin-binding transcription activator 1 mediates auxin signaling and responds to stresses in Arabidopsis. Planta. 2010;232(1):165-78. https://doi. org/10.1007/s00425-010-1153-6.

46. Galon Y, Snir O, Fromm H. How calmodulin binding transcription activators (CAMTAs) mediate auxin responses. Plant Signal Behav. 2010;5(10):1311-4. https://doi.org/10.4161/psb.5.10.13158.

47. Wang C, Shang J-X, Chen Q-X, Oses-Prieto JA, Bai M-Y, Yang Y, et al. Identification of BZR1-interacting proteins as potential components of the brassinosteroid signaling pathway in Arabidopsis through tandem affinity purification. Mol Cell Proteomics. 2013;12(12):3653-65. https://doi.org/10.1074/mcp.M113.029256.

48. Nie H, Zhao C, Wu G, Wu Y, Chen Y, Tang D. SR1, a calmodulin-binding transcription factor, modulates plant defense and ethylene-induced senescence by directly regulating NDR1 and EIN3. Plant Physiol. 2012;158(4): 1847-59. https://doi.org/10.1104/pp.111.192310.

49. Li X, Huang L, Zhang Y, Ouyang Z, Hong Y, Zhang H, et al. Tomato SR/CAMTA transcription factors SISR1 and SISR3L negatively regulate disease resistance response and SISR1L positively modulates drought stress tolerance. BMC Plant Biol. 2014;14(1):1-19. https://doi.org/10.1186/s12870-014-0286-3.

50. Pinsorn P, Oikawa A, Watanabe M, Sasaki R, Ngamchuachit P, Hoefgen R, et al. Metabolic variation in the pulps of two durian cultivars: unraveling the metabolites that contribute to the flavor. Food Chem. 2018;268:118-25. https://doi.org/10.1016/j.foodchem.2018.06.066.

51. Friedman H. Candidate genes to extend fleshy fruit shelf life. Plant Breeding Reviews. 2019;43:61-94.

52. Rahman H, Yang J, Xu Y-P, Munyampundu J-P, Cai X-Z. Phylogeny of plant CAMTAs and role of AtCAMTAs in nonhost resistance to Xanthomonas oryzae pv oryzae. Frontiers in plant science. 2016;7:177. https://doi.org/10.33 89/fpls.2016.00177.

53. Lescot $M$, Déhais $P$, Thijs $G$, Marchal $K$, Moreau $Y$, Van de Peer $Y$, et al. PlantCARE, a database of plant cis-acting regulatory elements and a portal to tools for in silico analysis of promoter sequences. Nucleic Acids Res. 2002;30(1):325-7. https://doi.org/10.1093/nar/30.1.325.

54. Higo K, Ugawa Y, Iwamoto M, Korenaga T. Plant cis-acting regulatory DNA elements (PLACE) database: 1999. Nucleic Acids Res. 1999;27(1):297-300. https://doi.org/10.1093/nar/27.1.297.

55. Xu H, Shi X, He L, Guo Y, Zang D, Li H, et al. Arabidopsis thaliana trihelix transcription factor AST1 mediates salt and osmotic stress tolerance by binding to a novel AGAG-box and some GT motifs. Plant Cell Physiol. 2018; 59(5):946-65. https://doi.org/10.1093/pcp/pcy032.

56. Ezer D, Shepherd SJ, Brestovitsky A, Dickinson P, Cortijo S, Charoensawan V, et al. The G-box transcriptional regulatory code in Arabidopsis. Plant Physiol. 2017;175(2):628-40. https://doi.org/10.1104/pp.17.01086.

57. Liu L, Xu W, Hu X, Liu H, Lin Y. W-box and G-box elements play important roles in early senescence of rice flag leaf. Sci Rep. 2016;6(1):1-9. https://doi. org/10.1038/srep20881.

58. Cruz AB, Bianchetti RE, Alves FRR, Purgatto E, Peres LEP, Rossi M, et al. Light, ethylene and auxin signaling interaction regulates carotenoid biosynthesis during tomato fruit ripening. Front Plant Sci. 2018;9:1370. https://doi.org/1 0.3389/fpls.2018.01370.

59. Özdemir is. Effect of light treatment on the ripening of banana fruit during postharvest handling. Fruits. 2016;71(2):115-22. https://doi.org/10.1051/ fruits/2015052.

60. Rouster J, Leah R, Mundy J, Cameron-Mills V. Identification of a methyl jasmonate-responsive region in the promoter of a lipoxygenase 1 gene expressed in barley grain. Plant J. 1997;11(3):513-23. https://doi.org/10.1046/ j.1365-313X.1997.11030513.X.

61. Goldsbrough AP, Albrecht H, Stratford R. Salicylic acid-inducible binding of a tobacco nuclear protein to a 10 bp sequence which is highly conserved amongst stress-inducible genes. Plant J. 1993;3(4):563-71. https://doi.org/1 0.1046/j.1365-313X.1993.03040563.x.

62. Shen $\mathrm{Q}, \mathrm{Ho}$ T. Functional dissection of an abscisic acid (ABA)-inducible gene reveals two independent $A B A$-responsive complexes each containing a $\mathrm{G}$ box and a novel cis-acting element. Plant Cell. 1995;7(3):295-307. https:// doi.org/10.1105/tpc.7.3.295.

63. Washida H, Wu C-Y, Suzuki A, Yamanouchi U, Akihama T, Harada K, et al. Identification of cis-regulatory elements required for endosperm expression of the rice storage protein glutelin gene GluB-1. Plant Mol Biol. 1999;40(1): 1-12. https://doi.org/10.1023/A:1026459229671.

64. Ulmasov T, Murfett J, Hagen G, Guilfoyle TJ. Aux/IAA proteins repress expression of reporter genes containing natural and highly active synthetic auxin response elements. Plant Cell. 1997;9(11):1963-71. https://doi.org/1 0.1105/tpc.9.11.1963.

65. Fujimoto SY, Ohta M, Usui A, Shinshi H, Ohme-Takagi M. Arabidopsis ethylene-responsive element binding factors act as transcriptional activators or repressors of GCC box-mediated gene expression. Plant Cell. 2000;12(3): 393-404. https://doi.org/10.1105/tpc.12.3.393.

66. Sessa G, Morelli G, Ruberti I. The Athb-1 and- 2 HD-zip domains homodimerize forming complexes of different DNA binding specificities. 
EMBO J. 1993;12(9):3507-17. https://doi.org/10.1002/j.1460-2075.1993.tb0602 5.X.

67. Trainotti L, Tadiello A, Casadoro G. The involvement of auxin in the ripening of climacteric fruits comes of age: the hormone plays a role of its own and has an intense interplay with ethylene in ripening peaches. J Exp Bot. 2007; 58(12):3299-308. https://doi.org/10.1093/jxb/erm178.

68. Khaksar G, Sangchay W, Pinsorn P, Sangpong L, Sirikantaramas S. Genomewide analysis of the Dof gene family in durian reveals fruit ripeningassociated and cultivar-dependent Dof transcription factors. Sci Rep. 2019; 9(1):1-13. https://doi.org/10.1038/s41598-019-48601-7.

69. Hobo T, Kowyama Y, Hattori T. A bZIP factor, TRAB1, interacts with VP1 and mediates abscisic acid-induced transcription. Proc Natl Acad Sci. 1999;96(26): 15348-53. https://doi.org/10.1073/pnas.96.26.15348.

70. Ruiz de Larrinaga L, Resco de Dios V, Fabrikov D, Guil-Guerrero JL, Becerril JM, García-Plazaola Jl, et al. Life after harvest: circadian regulation in photosynthetic pigments of rocket leaves during supermarket storage affects the nutritional quality. Nutrients. 2019;11(7):1519.

71. Duran-Soria S, Pott DM, Osorio S, Vallarino JG. Sugar Signaling During Fruit Ripening. Front Plant Sci. 2020;11. https://doi.org/10.3389/fpls.2020.564917.

72. Posé S, Paniagua C, Matas AJ, Gunning AP, Morris VJ, Quesada MA, et al. A nanostructural view of the cell wall disassembly process during fruit ripening and postharvest storage by atomic force microscopy. Trends Food Sci Technol. 2019;87:47-58. https://doi.org/10.1016/j.tifs.2018.02.011.

73. Teh BT, Lim K, Yong CH, Ng CCY, Rao SR, Rajasegaran V, et al. The draft genome of tropical fruit durian (Durio zibethinus). Nat Genet. 2017:49(11): 1633-41. https://doi.org/10.1038/ng.3972.

74. Forlani S, Masiero S, Mizzotti C. Fruit ripening: the role of hormones, cell wall modifications, and their relationship with pathogens. J Exp Bot. 2019; 70(11):2993-3006. https://doi.org/10.1093/jxb/erz112.

75. Lynch M, Conery JS. The evolutionary fate and consequences of duplicate genes. science. 2000;290(5494):1151-5.

76. Tanaka KM, Takahasi KR, Takano-Shimizu T. Enhanced fixation and preservation of a newly arisen duplicate gene by masking deleterious lossof-function mutations. Genet Res. 2009;91(4):267-80. https://doi.org/10.1017/ S0016672309000196.

77. Gao Q, Xiong T, Li X, Chen W, Zhu X. Calcium and calcium sensors in fruit development and ripening. Sci Hortic. 2019;253:412-21. https://doi.org/10.1 016/j.scienta.2019.04.069.

78. Reddy AS. Calcium: silver bullet in signaling. Plant Sci. 2001;160(3):381-404. https://doi.org/10.1016/S0168-9452(00)00386-1.

79. Liu J, Whalley HJ, Knight MR. Combining modelling and experimental approaches to explain how calcium signatures are decoded by calmodulinbinding transcription activators (CAMTA s) to produce specific gene expression responses. New Phytol. 2015;208(1):174-87. https://doi.org/1 $0.1111 / \mathrm{nph} .13428$

80. Cunha CP, Roberto GG, Vicentini R, Lembke CG, Souza GM, Ribeiro RV, et al. Ethylene-induced transcriptional and hormonal responses at the onset of sugarcane ripening. Sci Rep. 2017;7(1):1-18. https://doi.org/10.1038/srep433 64.

81. Hall AE, Findell JL, Schaller GE, Sisler EC, Bleecker AB. Ethylene perception by the ERS1 protein in Arabidopsis. Plant Physiol. 2000;123(4):1449-58. https:// doi.org/10.1104/pp.123.4.1449.

82. Tassoni A, Watkins $C B$, Davies PJ. Inhibition of the ethylene response by 1$M C P$ in tomato suggests that polyamines are not involved in delaying ripening, but may moderate the rate of ripening or over-ripening. J Exp Bot. 2006;57(12):3313-25. https://doi.org/10.1093/jxb/erl092.

83. Khaksar $\mathrm{G}$, Sirikantaramas $\mathrm{S}$. Auxin response factor $2 \mathrm{~A}$ is part of the regulatory network mediating fruit ripening through Auxin-ethylene crosstalk in durian. Front Plant Sci. 2020;11:543747. https://doi.org/10.3389/ fpls.2020.543747.

84. Su R, Hu H, Wu M, Guo W, Chen J, Wang C, et al. Isolation and characterisation of a UDP-glucose pyrophosphorylase gene associated with fruit ripening in autumn olive (Elaeagnus umbellata Thunb.). J Hortic Sci Biotechnol. 2013;88(5):617-23. https://doi.org/10.1080/14620316.2013.11513 015.

85. Wei Y, Hu W, Xia F, Zeng H, Li X, Yan Y, et al. Heat shock transcription factors in banana: genome-wide characterization and expression profile analysis during development and stress response. Sci Rep. 2016;6(1):1-11. https://doi.org/10.1038/srep36864.

86. Tan B, Lian X, Cheng J, Zeng W, Zheng X, Wang W, et al. Genome-wide identification and transcriptome profiling reveal that E3 ubiquitin ligase genes relevant to ethylene, auxin and abscisic acid are differentially expressed in the fruits of melting flesh and stony hard peach varieties. BMC Genomics. 2019;20(1):1-15.

87. Wu J, Fu L, Yi H. Genome-wide identification of the transcription factors involved in citrus fruit ripening from the transcriptomes of a late-ripening sweet orange mutant and its wild type. PLoS One. 2016;11(4):e0154330. https://doi.org/10.1371/journal.pone.0154330.

88. Frylinck $L$, Dubery IA. Protein kinase activities in ripening mango, Mangifera indica L., fruit tissue: III. Purification and characterisation of a calciumregulated protein kinase. Biochim Biophys Acta. 1998;1387(1-2):342-54.

89. Wang Q-H, Zhao C, Zhang M, Li Y-Z, Shen Y-Y, Guo J-X. Transcriptome analysis around the onset of strawberry fruit ripening uncovers an important role of oxidative phosphorylation in ripening. Sci Rep. 2017;7(1): 1-11. https://doi.org/10.1038/srep41477.

90. Houben M, Van de Poel B. 1-Aminocyclopropane-1-carboxylic acid oxidase (ACO): the enzyme that makes the plant hormone ethylene. Front Plant Sci. 2019;10:695. https://doi.org/10.3389/fpls.2019.00695.

91. Luo D, Ba L, Chen J, Lu W, Shan W, Kuang J. Characterization and expression analysis of banana MaTIFY1 transcription factor during fruit ripening. Acta Horticulturae Sinica. 2017;44(1):43-52.

92. Pua E-C, Lee $Y-C$. Expression of a ripening-related cytochrome P450 cDNA in Cavendish banana (Musa acuminata Cv. Williams). Gene. 2003;305(1):13340. https://doi.org/10.1016/S0378-1119(02)01237-4

93. Bisbis B, Delmas F, Joubès J, Sicard A, Hernould M, Inzé D, et al. Cyclindependent kinase (CDK) inhibitors regulate the CDK-cyclin complex activities in endoreduplicating cells of developing tomato fruit. J Biol Chem. 2006;281(11):7374-83. https://doi.org/10.1074/jbc.M506587200.

94. Pandey A, Misra P, Alok A, Kaur N, Sharma S, Lakhwani D, et al. Genome-wide identification and expression analysis of homeodomain leucine zipper subfamily IV (HDZ M) gene family from Musa accuminata. Front Plant Sci. 2016;7:20.

95. Cheng Y, JalalAhammed G, Yu J, Yao Z, Ruan M, Ye Q, et al. Putative WRKYs associated with regulation of fruit ripening revealed by detailed expression analysis of the WRKY gene family in pepper. Sci Rep. 2016;6(1):1-11. https:// doi.org/10.1038/srep39000.

96. Fu C, Chen H, Gao H, Lu Y, Han C, Han Y. Two papaya MYB proteins function in fruit ripening by regulating some genes involved in cell-wall degradation and carotenoid biosynthesis. J Sci Food Agric. 2020;100(12): 4442-8. https://doi.org/10.1002/jsfa.10484.

97. Asif MH, Lakhwani D, Pathak S, Bhambhani S, Bag SK, Trivedi PK. Genomewide identification and expression analysis of the mitogen-activated protein kinase gene family from banana suggest involvement of specific members in different stages of fruit ripening. Functional \& integrative genomics. 2014; 14(1):161-75. https://doi.org/10.1007/s10142-013-0349-9.

98. Xu Y, Xu H, Wall MM, Yang J. Roles of transcription factor SQUAMOSA promoter binding protein-like gene family in papaya (Carica papaya) development and ripening. Genomics. 2020;112(4):2734-47. https://doi. org/10.1016/j.ygeno.2020.03.009.

99. Y-C H, C-c F, Kuang J-f, J-y C, Lu W-j. Two banana fruit ripening-related $\mathrm{C} 2 \mathrm{H} 2$ zinc finger proteins are transcriptional repressors of ethylene biosynthetic genes. Postharvest Biol Technol. 2016;116:8-15.

100. Zhang Z, Li X. Genome-wide identification of AP2/ERF superfamily genes and their expression during fruit ripening of Chinese jujube. Sci Rep. 2018; 8(1):1-16. https://doi.org/10.1038/s41598-018-33744-w.

101. Du L, Ali GS, Simons KA, Hou J, Yang T, Reddy A, et al. Ca 2+/calmodulin regulates salicylic-acid-mediated plant immunity. Nature. 2009;457(7233): 1154-8. https://doi.org/10.1038/nature07612.

102. Decros G, Baldet P, Beauvoit B, Stevens R, Flandin A, Colombié S, et al. Get the balance right: ROS homeostasis and redox signalling in fruit. Front Plant Sci. 2019;10:1091. https://doi.org/10.3389/fpls.2019.01091.

103. Kumar V, Irfan M, Ghosh S, Chakraborty N, Chakraborty S, Datta A. Fruit ripening mutants reveal cell metabolism and redox state during ripening. Protoplasma. 2016;253(2):581-94. https://doi.org/10.1007/s00709-015-0836-z.

104. McAtee P, Karim S, Schaffer RJ, David K. A dynamic interplay between phytohormones is required for fruit development, maturation, and ripening. Front Plant Sci. 2013;4:79. https://doi.org/10.3389/fpls.2013.00079.

105. Jiang F, Lopez A, Jeon S, de Freitas ST, Yu Q, Wu Z, et al. Disassembly of the fruit cell wall by the ripening-associated polygalacturonase and expansin influences tomato cracking. Horticulture research. 2019;6(1):1-15.

106. Jin J, Zhang H, Kong L, Gao G, Luo J. PlantTFDB 3.0: a portal for the functional and evolutionary study of plant transcription factors. Nucleic Acids Res. 2014;42(D1):D1182-7. https://doi.org/10.1093/nar/gkt1016. 
107. Liu W, Xie Y, Ma J, Luo X, Nie P, Zuo Z, et al. IBS: an illustrator for the presentation and visualization of biological sequences. Bioinformatics. 2015; 31(20):3359-61. https://doi.org/10.1093/bioinformatics/btv362.

108. Hu B, Jin J, Guo A-Y, Zhang H, Luo J, Gao G. GSDS 2.0: an upgraded gene feature visualization server. Bioinformatics. 2015;31(8):1296-7. https://doi. org/10.1093/bioinformatics/btu817.

109. Bailey T, Boden M, Buske F, Frith M, Grant C, Clementi L, Noble W: MEME Suite: Tools for motif discovery and searching. Nucleic Acids Res. 2009; 37(Suple.2):W202-8.

110. Tamura K, Peterson D, Peterson N, Stecher G, Nei M, Kumar S. MEGA5: molecular evolutionary genetics analysis using maximum likelihood, evolutionary distance, and maximum parsimony methods. Mol Biol Evol. 2011;28(10):2731-9. https://doi.org/10.1093/molbev/msr121.

111. Yang S, Zhang X, Yue J-X, Tian D, Chen J-Q. Recent duplications dominate NBS-encoding gene expansion in two woody species. Mol Gen Genomics. 2008;280(3):187-98. https://doi.org/10.1007/s00438-008-0355-0.

112. Suyama M, Torrents D, Bork P. PAL2NAL: robust conversion of protein sequence alignments into the corresponding codon alignments. Nucleic acids research. 2006;34(suppl_2):W609-12.

113. Die JV, Gil J, Millan T. Genome-wide identification of the auxin response factor gene family in Cicer arietinum. BMC Genomics. 2018;19(1):1-15. https://doi.org/10.1186/s12864-018-4695-9.

114. Juretic N, Hoen DR, Huynh ML, Harrison PM, Bureau TE. The evolutionary fate of MULE-mediated duplications of host gene fragments in rice. Genome Res. 2005;15(9):1292-7. https://doi.org/10.1101/gr.4064205.

115. Li W-H, Gojobori T. Rapid evolution of goat and sheep globin genes following gene duplication. Mol Biol Evol. 1983; (1):94-108. https://doi.org/1 0.1093/oxfordjournals.molbev.a040306.

116. Sirikantaramas S, Sangpong L, Khaksar G, Pinsorn P, Oikawa A, Sasaki R, et al. Assessing dynamic changes of taste-related primary metabolism during ripening of durian pulp using metabolomic and transcriptomic analyses Front Plant Sci. 2021;12:1198. https://doi.org/10.3389/fpls.2021.687799.

117. Chong J, Soufan O, Li C, Caraus I, Li S, Bourque G, et al. MetaboAnalyst 4.0 towards more transparent and integrative metabolomics analysis. Nucleic Acids Res. 2018;46(W1):W486-94. https://doi.org/10.1093/nar/gky310.

118. Usadel B, Poree F, Nagel A, Lohse M, Czedik-Eysenberg A, Stitt M. A guide to using MapMan to visualize and compare Omics data in plants: a case study in the crop species, maize. Plant Cell Environ. 2009;32(9):1211-29. https://doi.org/10.1111/j.1365-3040.2009.01978.x.

119. Livak K, Schmittgen T: Analysis of relative gene expression data using realtime quantitative PCR and the $2-\Delta \Delta C T$ method. methods 25: 402-408. Find this article online 2001.

\section{Publisher's Note}

Springer Nature remains neutral with regard to jurisdictional claims in published maps and institutional affiliations.

Ready to submit your research? Choose BMC and benefit from:

- fast, convenient online submission

- thorough peer review by experienced researchers in your field

- rapid publication on acceptance

- support for research data, including large and complex data types

- gold Open Access which fosters wider collaboration and increased citations

- maximum visibility for your research: over $100 \mathrm{M}$ website views per year

At $\mathrm{BMC}$, research is always in progress.

Learn more biomedcentral.com/submissions 